\title{
Die Nacht des Kindes in seinem Nest in Dendara
}

Inhalt:

I. Einleitung

II. Dokumentation

III. Die Orientierung des großen Hathortempels und des kleinen Isistempels

IV. Die Geburt der Isis-Sothis als dunkelrote Frau (st kmt dšrt)

V. Das Herbeibringen der šs $d$-Binde

VI. Die Inschrift D IV, 60, 11-3 - eine genaue Zeitangabe des Siriusaufgangs?

VII. Die Darstellung auf der Westwand des Schatzhauses ( $p r-\underline{b l}$; ; st nfrt = Raum Q)

VIII. Die Neujahrsbezeichnung „Horn des Steinbocks” ( $b$ ni3w)

Anhang: Die Identifikation des Tiers $t 3 w$ als ghs: „Dorkasgazelle”

\section{Einleitung}

Diese etwas merkwürdige Tagesbezeichnung, ausführlicher meist der Tag der Nacht des Kindes in seinem Nest ( $b r w$ grh $n h n$ m šs.f) erschien $\mathrm{m}$. W. das erste Mal 1877 in der ägyptologischen Fachliteratur, der Autor J. Dümichen übersetzte sie auf Anhieb richtig mit „5. Epagomenentag“ " ${ }^{1}$. Im gleichen Jahr veröffentlichte jedoch H. Brugsch seine Studie über die griechisch-römischen Festkalender, in der er auf S. 21 eine Ergänzung vornahm ${ }^{2}$. Er liest dort an einer seiner Ansicht nach zerstörten Stelle: „Schalttag (4). In dieser schönen Zeit der Nacht des Säuglings in seiner Wiege ...", die Gründe für diese Emendierung legt er an einer anderen Stelle dar ${ }^{3}$ :

„Über den in den tentyritischen Inschriften so häufig erwähnten Ausdruck: ... „die Zeit (wörtlich: der Tag) der Nacht des Kindes in seiner Wiege" wissen wir nur so viel, daß der gemeinte Tag zu den 5 Schalttagen am Schlusse des Jahres gehörte. Die bestimmtere Angabe ist leider an der betreffenden Stelle in der Festkalender-Liste des Tempels von Dendera zerstört. $\mathrm{Da}$ aber nach sonst erhaltenen Verzeichnissen der 4. Schalttag als Tag der Geburt der Göttin

\footnotetext{
${ }^{1}$ Baugeschichte des Denderatempels und Beschreibung der einzelnen Theile des Bauwerkes, Strassburg, 1877, 28 mit Bezug auf die Zeile 15 der Tafel XXXVIII. - Alle Abkürzungen folgen den Regeln des Lexikons der Ägyptologie (Hrgb. W. Helck und W. Westendorf), Bd. VII, Wiesbaden, 1989, IX-XXXVIII.

${ }^{2}$ Drei Fest-Kalender des Tempels von Apollinopolis magna in Ober-Aegypten, Leipzig, 1877.

${ }^{3}$ Brugsch, Thes., 97-8.
} 
Isis notiert ist, so dürfen wir wohl mit Recht die Zahl 4 an der unlesbaren Stelle des Kalenders substituieren."

Dieser (Fehl)Überlegung, die den Weg zum Verständnis zahlreicher Dendaratexte über den Neujahrstag verbaut, sind seither alle Ägyptologen gefolgt. Alliot bietet bei seiner Neuedition des Festkalenders in Dendara im hieroglyphischen Text die gleiche Ergänzung wie Brugsch, in seiner Übersetzung fehlt jedoch die Markierung der Ergänzung ${ }^{4}$. Auf Alliot berufen sich wieder andere ${ }^{5}$, ein Vorstoß des Verfassers, es handle sich doch um den 5. Epagomenentag ${ }^{6}$, hat anscheinend nur das Gegenteil bewirkt. Entweder wird unter Berufung auf eben dieses Buch behauptet, es handle sich doch um den 4. Epagomenentag ${ }^{7}$ oder es wird gerade dieses Phänomen in einer längeren Polemik als Beispiel dafür herangezogen, wie wenig Ahnung doch der Verfasser von den „Egyptian religious mental constructs“ hätte und wie wichtig es sei, aufmerksam die ägyptischen Texte zu lesen und nach ihrem sog. Sitz im Leben zu suchen ${ }^{8}$.

Um den Sachverhalt noch einmal völlig zweifelsfrei zu formulieren: Für den Geburtstag der Isis gibt es zwei unterschiedliche Traditionen, die miteinander nichts zu tun haben. Handelt es sich um die Geburtstage der fünf Kinder der Nut an den fünf Epagomenen, so fällt der Geburtstag der Isis auf den 4. Epagomenentag. Handelt es sich dagegen um die Göttin IsisSothis, so fällt ihre Geburt per definitionem in die letzte Stunde des alten Jahres, d. h. in die Nacht des 5. Epagomenentages, kurze Zeit danach beginnt mit Sonnenaufgang der Neujahrstag.

\section{Dokumentation}

Im folgenden wird die Nacht des Kindes in seinem Nest an Hand der mir bekannten 62 publizierten Textstellen aus Dendara ${ }^{9}$ untersucht. Hierzu ist zu sagen, daß es mit Sicherheit noch weitere Belege in den nicht veröffentlichten Tempelteilen und in dem kleinen Isistempel auf der Südseite des Hathortempels gibt, gleichzeitig das Material aber so homogen ist, daß auch die noch unbekannten Stellen nicht allzuviel Neues bringen dürften. 9 der 62 Stellen werden im Anschluß an die Dokumentation angeführt, da sie Besonderheiten enthalten, die weitergehende Überlegungen ermöglichen.

Die (vermeintliche) Ausnahme sei vorweggestellt: In Urk. VI, 143,18 und 145,4 wird der Tag $n h n$ s š̌ff als Name des 4. Epagomenentages angeführt. Die Erklärung ist einfach: In dieser Handschrift sind die Namen des 1. und 2. sowie des 4. und 5. Epagomenentages miteinander vertauscht, die drei anderen Handschriften ${ }^{10}$ bieten die richtige Verteilung; die Verbindung des hrw grḥ nh̆n $m$ š̌.f zum 5. Epagomenentag und speziell zum Neujahrstag wird das Folgende verdeutlichen.

Die nachstehenden Texte, deren Vielzahl die Bedeutung dieses Tages für den Kult von Den-

\footnotetext{
${ }^{4}$ M. Alliot, Le culte d'Horus à Edfou au temps des Ptolémées, BdE 20, Le Caire, 1949, 242 und 249 (Le $4{ }^{\mathrm{e}}$ des „,cinq jours de l'an“... c'est la belle journée de la „nuit de l'Enfant dans son nid“).

${ }^{5}$ Daumas, Mammisis, 32-3 mit Anm. 2 auf S. 33. Derselben Ansicht C. Husson, L'offrande du miroir dans les temples égyptiens de l'époque gréco-romaine, Lyon, 1977, 115, 261 und 266, Anm. 57.

${ }^{6}$ Studien zur ägyptischen Astronomie, ÄA 49, Wiesbaden, 1989, 4-5.

${ }^{7}$ Cauville, BIFAO 90, 1990, 93 mit Anm. 22.

${ }^{8}$ Spalinger in: OLZ 87, 1992, 24 (ohne Belege), ders. in: BES 10, 1989/90, 137, Anm. 2 (2).

${ }^{9}$ Fast alle Zitate für hrw grḥ nhn $m$ š̌.f stammen aus Dendara, aus Philae kenne ich nur Junker, Philae I, 108 (vgl. zum Pylon und seiner Beziehung zum Aufgang der Sothis Leitz, op. cit., 59-60), in Edfu findet sich entgegen Spalingers Behauptung zumindest in Chassinat's Edition kein Beleg.

${ }^{10}$ Angeführt bei Leitz, op. cit., 4-5 und in der kommenden Arbeit über die Tagewählkalender im Kapitel über die Epagomenen.
} 
dara herauszustellen vermag, behandeln in immer neuen Varianten alle das gleiche Thema, die Geburt der Isis-Sothis in I3t-dit, dem kleinen Tempel in der (geographischen) Südwestecke des großen Tempels, d. h., da die Himmelsrichtungen in den Inschriften um $90^{\circ}$ verschoben sind, in der Nordwestecke des Tempels ${ }^{11}$. Die meisten, jedoch nicht alle Erwähnungen der „Nacht des Kindes in seinem Nest" stehen auch auf einer Süd- oder Westwand. In der überwiegenden Anzahl der Fälle wird gesagt, daß die Isis geboren wird; das Verb $m s i$ ist dabei das, was astronomisch „heliakisch aufgehen“ bedeutet ${ }^{12}$, in einigen Texten wird dabei die Sothis explizit genannt ${ }^{13}$. In anderen Texten gilt sie als Bringerin des Überschwemmungswassers ${ }^{14}$, bisweilen wird das Geschehnis auch direkt auf das Neujahrsfest (wpt-rnpt oder $h b w p t$-rnpt) gelegt ${ }^{15}$, ein Fest, bei dem zwei kalendarische Ereignisse gefeiert wurden. Zum einen war dies in der Morgendämmerung, etwa 40 min. vor Sonnenaufgang, kalendarisch noch der 5. Epagomenentag, der Nacht des Kindes in seinem Nest, der heliakische Aufgang des Sirius, zum anderen, bei Sonnenaufgang, der Beginn des neuen Kalenderjahres, der I. 3ht 1. Der jugendliche Sonnengott ist dabei das Kind in seinem Nest, der Falke ${ }^{16}$, der sich bei Sonnenaufgang am Neujahrstag das erste Mal zum Himmel erheben wird, mit dem dann der König gleichgesetzt werden kann ${ }^{17}$. Die beiden Himmelskörper (b3yty), von denen hierbei des öfteren die Rede ist ${ }^{18}$, sind der Sirius und die Sonne, nicht wie sonst oft Sonne und Mond ${ }^{19}$, dasselbe ist gemeint, wenn von den beiden Augen der Göttin die Rede ist, das Öffnen bezeichnet dabei jeweils den Aufgang des Himmelskörpers.

Dabei gilt die Sothis als rechtes Auge, die Morgensonne als linkes Auge, das Überstrahlen der viel lichtschwächeren Sothis durch die Sonne wird die Vereinigung des rechten mit dem linken Auge genannt (z. B. D VII, 179,1-2). Die Verteilung nach rechts und links entspricht dabei durchaus den natürlichen Verhältnissen, wenn man berücksichtigt, daß die Himmelsrichtungen in den Inschriften um $90^{\circ}$ gedreht sind. D. h. der geographische Osten wird zum Süden (rsy), die Sothis wird bei ihrem heliakischen Aufgang etwa $19^{\circ 20}$ südlich des geographischen Ostpunktes sichtbar, steht also in ägyptischer Terminologie im Westen des Südpunktes und

${ }^{11}$ Vgl. hierzu F. Daumas, Dendara et le temple d'Hathor, RAPH 29, Le Caire, 1969, X; ders., Mammisis, 30-1 (mit Anm. 2 auf S. 31), der Text der Westkrypte Nr. 2 ist mittlerweile publiziert in D VI, 156.

${ }^{12}$ So H. O. Lange/O. Neugebauer, Papyrus Carlsberg Nr. 1, Kopenhagen, 1940, 57-63 in ihrer Analyse zu Kapitel E des Papyrus, die auch noch durch die Kapitel H und L unterstützt wird. Die Bemerkung von Krauss in BSEG 14, 1990, 55, Anm. 34, hierin lediglich einen „Vorschlag“ zu sehen, kommt doch etwas überraschend, was sind denn die Alternativen? Doch wohl kaum sein eigener Vorschlag, den Terminus mst für den Aufgang des Sterns im Horizont zu reservieren, ein Vorgang, der mit bloßem Auge nicht zu beobachten ist und dieses Ereignis dann vom Sichtbarwerden des Sterns etwa 13 min. später, eben dem heliakischen Aufgang zu unterscheiden.

${ }^{13}$ Text (2); (4); (8); (19).

${ }^{14}$ Text (4); (8); (38); (43).

${ }^{15}$ Text (2) und (66); meist wird nur allgemein gesagt, daß es sich um ein großes Fest des ganzen Landes handele.

${ }^{16}$ Eine andere Bezeichnung für den gerade flügge gewordenen Falken ist $\check{s} r$ (hr ), vgl. D II, 110,6-7 (gehört zu Text (12)), wo der König den Beinamen ,̌̌r , der Sohn des $r n p t n f r t$ " trägt, die Beziehung zum Neujahr ist durch den Titel gegeben (vgl. dazu unten Abschnitt V).

${ }^{17}$ Siehe z. B. Junker, Philae I, 212 [Gleichsetzung von König und Horus, dem Kind in seinem Nest]. Vgl. auch Edfou I², 67,15-7 [Milchopfer für das Kind in seinem Nest]; IV, 272,11-2 [in einem Milchopfer: „Ptolemaios, der gute Gott, das Kind in Chemmis, der Sproß der Wadjet, die das Kind in seinem Nest beschützt"], ähnlich D I, 5,12-3 und II, 205,15. Für das Kind in seinem Nest werden die Annalen bereits auf der Geburtsstätte geschrieben (Daumas, Mammisis de Dendara, 137,1) und das Regierungsamt (i3wt) wird ihm übergeben (Daumas, Mam., 125,4-5).

${ }^{18}$ Text (8); (35); (40).

19 Z. B. Edfou III, 268,16-7.

${ }^{20}$ Rechnung nach P. V. Neugebauer, Tafeln zur astronomischen Chronologie III, Leipzig, 1922, \17 (bei einer Höhe von $2^{\circ} .3$ über dem Horizont). 


\section{Die ideellen Himmelsrichtungen in Dendara}

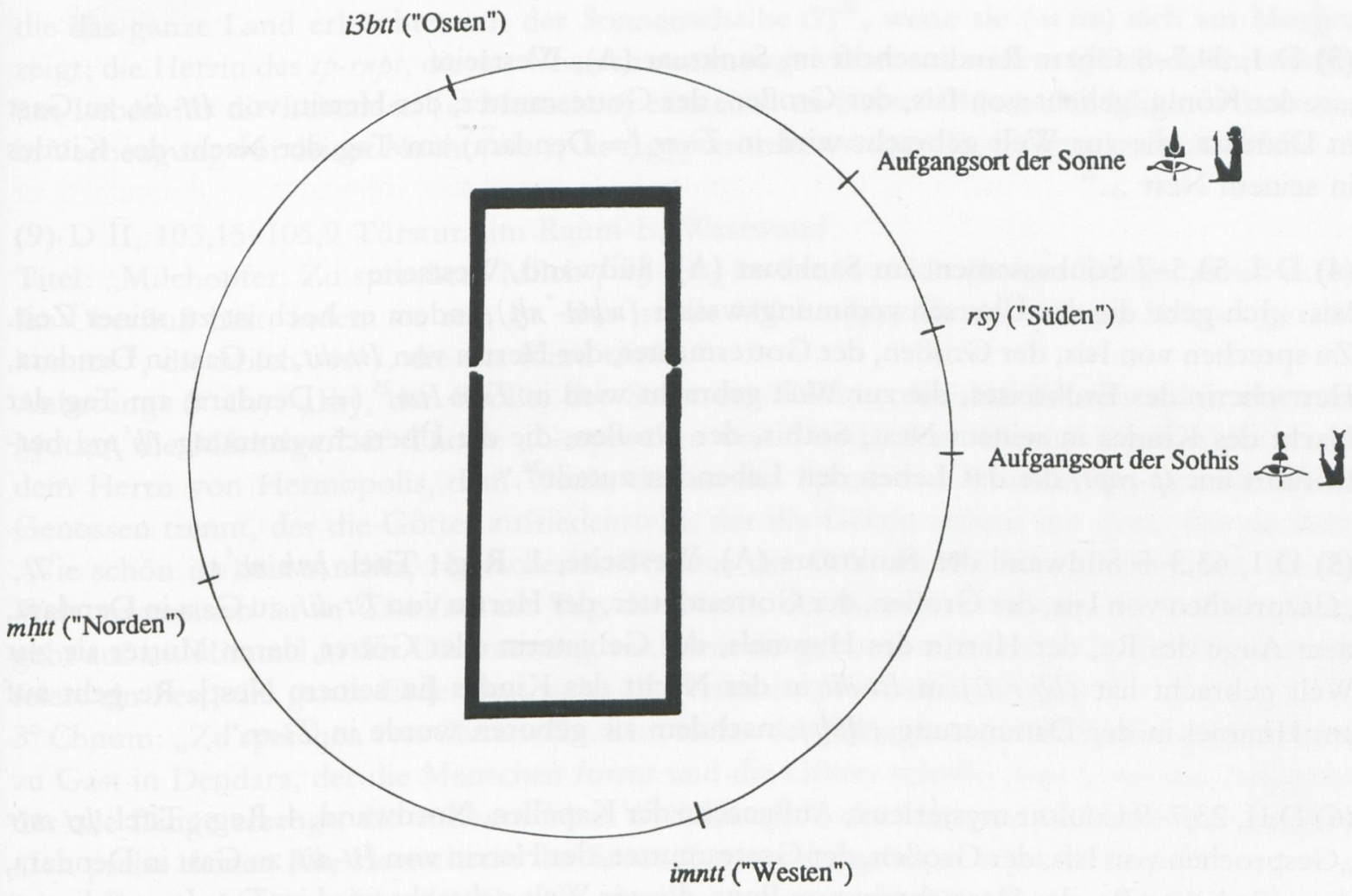

Abb. 1

kann so gut imnt: „westliches/rechtes Auge“ genannt werden, die Sonne dagegen geht rund $25^{\circ 21}$ nördlich des geographischen Ostpunktes auf, steht mithin in der Terminologie von Dendara im Osten des Südpunktes und wird folgerichtig ỉbt: „östliches/linkes Auge“ genannt, die beigefügte Abbildung möge dies noch einmal verdeutlichen.

(1) D(endara) I, 7,2-3 Fassade des Sanktuars (A), 1. Reg. Westseite; Titel: dw $n \underline{s} \underline{\mathbf{t}}$ sp 4 „Gesprochen von Isis, der Großen, der Gottesmutter, Der Herrin von I3t-dit, zu Gast in Dendara, die neugeboren wird (nhh $)$ in Ntry (Dendara) in der Nacht des Kindes in seinem Nest."

(2) D I, 20, ult. - 21,2 Westlicher Türpfosten zum Sanktuar (A) „Isis, die Große, die Gottesmutter, die Herrin von I3t-dit, zu Gast in Dendara, die Herrin des $t p-r n p t^{22}$, die Gebieterin der $\check{s} m 3 y w$-Dämonen ${ }^{23}$, die aufgeht am Neujahrsfest (wpt-rnpt), um ein schönes Jahr zu eröffnen, Imnt in Theben, Mnbyt in Esna, Rnpt (Jahr) ist ihr Name in Memphis,

\footnotetext{
${ }^{21}$ Rechnung nach Neugebauer, op. cit., $\int 18$ (genauer $25^{\circ} .2$ ).

${ }^{22} \mathrm{Vgl}$. Spalinger in SAK 17, 1990, 290. Zumindest hier ist damit der Jahresanfang gemeint, vgl. Text (2), (4) und (32).

${ }^{23}$ Eine Göttergruppe, die oft zusammen mit den ḩ3tyw-Dämonen auftritt und für die Verbreitung der i3dt-rnpt insbesondere zur Zeit der Epagomenen verantwortlich ist (z. B. pJumilhac XVIII, 5-9), siehe dazu ausführlich den Exkurs über die ḩstyw-Dämonen in meiner Arbeit über die Tagewählerei.
} 
Sothis in Elephantine ${ }^{24}$, Hededet in Edfu, Nechbet, die Vornehme, die Herrin von Hierakonpolis, Iwnt in Dendara, die Tochter der Nut, die zur Welt gebracht wurde in I3t-dit an diesem schönen Tag des Kindes in seinem Nest ..."

(3) D I, 34,7-8 Obere Randinschrift im Sanktuar (A), Westseite

„... der König, geliebt von Isis, der Großen, der Gottesmutter, der Herrin von I3t-dit, zu Gast in Dendara, die zur Welt gebracht wird in T3-rr (=Dendara) am Tag der Nacht des Kindes in seinem Nest ..."

(4) D I, 54,5-7 Soubassement im Sanktuar (A), Südwand, Westseite

Isis: „Ich gebe dir das Überschwemmungswasser ( $\left.w h m-{ }^{-} n h\right)$, indem es hoch ist zu seiner Zeit. Zu sprechen von Isis, der Großen, der Gottesmutter, der Herrin von I3t-dit, zu Gast in Dendara, Herrscherin des Erdkreises, die zur Welt gebracht wird in T3- $n-I_{t m}{ }^{25}$ (= Dendara) am Tag der Nacht des Kindes in seinem Nest, Sothis, der Großen, die die Überschwemmung ( $\left.b^{c} p y\right)$ herbeiführt am tp-rnpt, die das Leben den Lebenden zuteilt ${ }^{26}$.“

(5) D I, 63,3-5 Südwand des Sanktuars (A), Westseite, 1. Reg.; Titel: ḅnk $m 3^{c} t$ "Gesprochen von Isis, der Großen, der Gottesmutter, der Herrin von I3t-dit, zu Gast in Dendara, dem Auge des Re, der Herrin des Himmels, der Gebieterin aller Götter, deren Mutter sie zur Welt gebracht hat $(b \underline{b} r$ ts) in I3t-dit in der Nacht des Kindes [in seinem Nest]. Re geht auf am Himmel in der Dämmerung (3h3h), nachdem sie geboren wurde in T3-rr.“

(6) D II, 23,7-9 Couloir mystérieux, Außensẹte der Kapellen, Nordwand, 4. Reg.; Titel: irt sntr „Gesprochen von Isis, der Großen, der Gottesmutter, der Herrin von I3t-dit, zu Gast in Dendara, dem göttlichen $\mathrm{Ba}$, der Herrscherin von Punt, die zur Welt gebracht wird in T3-n-Itm an diesem schönen Tag der Nacht des Kindes in seinem Nest. Der Aufgehende am Himmel geht auf, nachdem sie ihr Auge geöffnet hat ...? ... dieses Land in der Dämmerung ${ }^{27}$.

(7) D II, 96,15 Türsturz zu Raum E, ob. Reg.

Beischrift zu einem falkenköpfigen Gott mit Sonnenscheibe: „Zu sprechen von dem Gott in der Nacht des Kindes in seinem Nest. Ich preise deinen (fem.) Ka im Augenblick (?) ${ }^{28}$ des Erkennens."

(8) D II, 98,2-99,3 Nördlicher Türpfosten zu Raum E

„Zu sprechen: Lobpreis sei dir, Lobpreis sei deinem Ka, (o) Isis, Gottesmutter, Herrin von I3t-dit, zu Gast in Dendara. Das ist die Uranfängliche ( $\left.\tilde{s}^{3} t\right)$, die zuerst vor Re Entstandene, die Einzigartige, die Gebieterin der Götter, die von ihrer Mutter zur Welt gebracht wird (3h3h) (? $)^{29}$ in T3-rr an diesem schönen Tag der Nacht des Kindes in seinem Nest. Die beiden

\footnotetext{
${ }^{24}$ Der Hintergrund dürfte sein, daß der allgemeine Kalenderbezugspunkt Memphis war; die Verbindung von Sothis und Elephantine beruht auf der zu dieser Zeit weit verbreiteten Gleichsetzung von Sothis und Satis, siehe z. B. D. Valbelle, Satis et Anoukis, Mainz, 1981, 157 index s. v. Sothis.

${ }^{25}$ Vermutlich so zu lesen gegen Gauthier, DG VI, 23, vgl. die volleren Schreibungen in D I, 68,12-3; VI, 4,1; 110,7-8.

${ }^{26}$ Ausführlicher geschrieben in D II, 99,2.

${ }^{27}$ Für ḋsw vgl. Hornung in: ZÄS 87, 1962, 119.

${ }^{28}$ hn, vgl. Wb III, 289, d. h. wörtlich „,bei der Angelegenheit, im Falle des Erkennens“ (sc. der Sothis im Horizont?).

${ }^{29}$ Lesung nicht sicher, Übersetzung nach dem Determinativ und dem Zusammenhang.
} 
Himmelskörper (b3yty) gehen auf am Himmel in der Dämmerung, während du deine beiden Augen öffnest in T3-n-Itm. ... Das ist die Herrscherin über das ganze Land, die Fürstin auf dem Erdkreis, Rat, die erscheint am tp-rnpt am Himmel als Sothis, die Gebieterin der Sterne, die das ganze Land erleuchtet mit der Sonnenscheibe (?) ${ }^{30}$, wenn sie $(=i t n)$ sich am Morgen zeigt; die Herrin des tp-rnpt, die das Überschwemmungswasser aus seinem Quelloch herausholt, um Leben für die Lebenden (= Menschen) entstehen zu lassen. Männer und Frauen kommen in Verbeugung vor deiner Macht (b3w), die Ägypter stehen wegen dir mit geneigtem Kopf da ... “

(9) D II, 103,15-105,9 Türsturz im Raum E, Westwand

Titel: „Milchopfer. Zu sprechen: Milch ( $n h$-w2ss) ist das, was ich dir hier bringe, damit du an ihr Überfluß hast, indem sie rein ist. Das ist die Milch (ḅ̣), die aus deinem Körper herauskommt ${ }^{31}$, die Milch (bnr), die das Kind trinkt."

Anbetung: $1^{\circ}$ Ihy: „Ihy, der Große, der Sohn der Hathor, der das Sistrum spielt für seine Mutter, die Mächtige.“ $2^{\circ}$ Thoth: „Gesprochen von Thoth, dem überaus Großen ('3 '3 wr), dem Herrn von Hermopolis, dem Wezir, der an der Spitze von T3-n-Itm ist, der die beiden Genossen trennt, der die Götter zufriedenstellt, der die Göttin erfreut mit dem, was sie liebt: ,Wie schön ist dein Antlitz, Herrscherin, Tochter des Geb, die die Nut geboren hat, die zur Welt gekommen ist in T3-n-Itm am Tag der Nacht des Kindes in seinem Nest. Dein Vater geht auf am Himmel in der Dämmerung, nachdem du geboren wurdest in I3t-dit. Der Himmel feiert ein Fest, die ganze Erde freut sich, die Neunheit preist dein Antlitz. “

$3^{\circ}$ Chnum: „Zu sprechen von Chnum-Re, dem Herrn des Kataraktengebietes, dem großen Gott, zu Gast in Dendara, der die Menschen formt und die Götter schafft, dem Vater am Urbeginn, der alle Dinge erzeugt, die vollkommene ' $\underline{c}^{e}$-Schlange ${ }^{32}$, der Herr der Geburtsstätte (mshnt): „Ich preise deinen Ka, Herrscherin der Stätten, wenn du geboren wirst in I3t-dit. “ ...

Isis: „Gesprochen von Isis, der Großen, der Gottesmutter, der Herrin von I3t-dit, zu Gast in Dendara, der Fürstin, die geboren wird in der st-mshnt (= Raum E), der dunkelroten Frau ${ }^{33}$, die das Leben ergreift ( $\underline{b} n m t-{ }^{-} n h$ ), die den ins-Stoff liebt, die vollkommen Jugendliche, süß an Liebe, ohne daß es eine andere gibt, die ihr gleichkommt am Morgen. Die Götter preisen sie jeden Tag, und die Göttinnen jubeln ihr zu."

(10) D II, 105,11-2 Türpfosten auf der Westwand des Raumes E, Nordseite „Zu sprechen: Gegrüßest seist du, Herrscherin, Tochter des Geb, Isis, Große, Gottesmutter, die zur Welt kommt in T3-n-Itm am Tag der Nacht des Kindes in seinem Nest. Re geht auf am Himmel in der Dämmerung, nachdem sie herausgekommen ist aus dem Mutterleib in T3-rr ..."

(11) D II, 108,5-8 Obere Randinschrift in Raum E, Südseite „Er (= der König) hat diese schöne st-mshnt gebaut, die fest gegründet ist auf ihrem Fundament seit Urzeiten, die Stätte, wo sie geboren wird von ihrer Mutter am Tag der Nacht des Kindes

\footnotetext{
$30 \mathrm{~m}$ itn?

${ }^{31}$ Anspielung auf das Licht ( $\left.\underline{b} \underline{d}\right)$ der aufgehenden Sothis.

32 'ḅe nfr, der Agathodaimon, siehe Kees, ZÄS 57, 1921, 121 ff.; J. Quaegebeur, Le dieu égyptien Shai, OLA 2, Leuven, 1975, 112, 140-1, 152-5 und F. Labrique, Stylistique et théologie à Edfou, OLA 51, Leuven, 1992, 254, Anm. 1230 und 1237; für seine Verbindung zum Neujahr G o y on in: Hommages à François Daumas, Montpellier, 1986, 336 mit Anm. 26 auf S. 340. Vgl. zu Chnum die Beischrift zur 2. Vignette in Kol. VIII (unten) im pJumilhac, wo bei einem widderköpfigen (!) Gott, auf dessen Haupt eine Schlange ist, steht: „Das ist die “ $\not b^{\prime}-S c h l a n g e ~ d e r$ Nekropole. Sie hat das Licht denen gegeben, die in der Dat sind.“

${ }^{33}$ st kemt ďrrt, siehe hierzu ausführlich weiter unten Abschnitt IV.
} 
in seinem Nest. Es geht das Kind (hy) am Himmel auf in der Dämmerung, nachdem sie ihre Augen geöffnet hat in T3-rr ..."

(12) D II, 110,15-6 Raum E, Nordwand, 1. Reg.

Titel $^{34}$ : Herbeibringen der š́d-Binde ${ }^{35}$ des schönen Jahres (rnpt nfrt $)^{36}$ für Isis, die Große, die Gottesmutter, die Herrin von 13t-dit, zu Gast in Dendara, die Tochter des Geb in der st-mshnt. Bereiten des Weihrauchs für das schöne Jahr [...].“

Göttliche Randzeile: „Die Königin von Ober- und Unterägypten, die zur Welt gebracht wird in I3t-dit, um Herrscherin des Erdkreises zu sein. Es geht der Aufgehende ${ }^{37}$ auf am Himmel in der Dämmerung am Tag der Nacht des Kindes in seinem Nest. Ihre Jahre sind $n \underline{h \underline{h}}$ und $\underline{d} t$. Isis, die Gottesmutter.“

(13) D II, 115,11-4 Raum E, Ostwand, Nordseite, 2. Reg.; Titel: Stoffopfer für seine Mutter, die Mächtige (wsrt)

Isis: „Gesprochen von Isis, der Großen, der Gottesmutter, der Herrin von I3t-dit, zu Gast in Dendara, mit leuchtender Brust, Herrin des Schmucks, der Schönen, der Vollkommenen, die den Stoff trefflich macht, Herrin der Furcht wegen der Furcht vor Sachmet ${ }^{38}$, das ist die Königin der beiden Länder, die Fürstin auf dem Erdkreis. Sndm $t^{39}$ hat sich niedergelassen auf ihrem Thron als große Herrscherin von Ägypten beim Bestrahlen ihres Tempels, beim Erleuchten dieses Landes, beim Erhellen der Gesichter mit ihren Strahlen. Sie ist eine schöne Katze $^{40}$ in der Nacht des Kindes in seinem Nest. Sie erhellt die beiden Länder und die Ufer mit ihren Glanzaugen (3hty).“

(14) D II, 118,9-10 Raum E Ostwand, Nordseite, 3. Reg. ${ }^{41}$; Titel: Spiegelopfer (ḥnk wnty-ḥr) Isis: „Gesprochen von Isis, der Großen, der Gottesmutter, dem Auge des Re, der Herrscherin des Himmels, der Gebieterin aller Götter, die zur Welt gebracht wird in T3-rr am Tag der Nacht des Kindes in seinem Nest, die Schöngesichtige, die Beliebte, die das Land mit Goldstaub erfüllt."

(15) D II, 122,7-10 Raum E Ostwand, Südseite, 1. Reg.; Titel: ḅnk $m 3^{c} t$

Isis: „Gesprochen von Isis, der Großen, der Gottesmutter, der Herrin von I3t-dit, zu Gast in Dendara, deren Mutter sie geboren hat in T3-rr, der Jugendlichen, der Schönen, der Herrin der Göttinnen, der Sonnenscheibe (itnt), die veranlaßt, daß die Sonnenscheibe (itn) strahlt: „Ich lasse Gerechtigkeit ( $g s-\underline{d} b)$ entstehen im Land zur Zeit deiner Majestät.“ Die Fürstin der Vorfahren, die die Abgaben aus dem Erdkreis einsammelt, deren Mutter Nut sie geboren hat in Hwt-Nwt (=Dendara) am Tag der Nacht des Kindes in seinem Nest. Sie hat den Göttern und Göttinnen Befehle erteilt. Isis, die Große, die Gottesmutter.“

\footnotetext{
${ }^{34}$ Eine vollständige Übersetzung des ganzen Tableaus bei E1-Kordy in: Fs Gutbub, 127-8.

${ }^{35}$ Siehe hierzu weiter unten Abschnitt V.

${ }^{36} \mathrm{Vgl}$. hierzu die Invocations à la Bonne Année, Ph. Germond, AH 11, Genf, 1986.

${ }^{37}$ Gemeint ist Re, vgl. das Determinativ von $w b n w$.

${ }^{38}$ Ein Stoff (vgl. D II, 102,3-4; 120,6, 149, vorletzte Zl. und Wb IV, 185,8), dessen Farbe rot (ins) war, D IV, 144,10 (Goyon in: Livre du centenaire, MIFAO 104, Le Caire, 1980, 25), vgl. dazu unten Abschnitt IV.

${ }^{39} \mathrm{Zu}$ dieser Göttin siehe Gutbub in Mel. Masp. I, 4,68-9.

${ }^{40}$ Vgl. Wb II, 42,7 (z. B. D II, 44,1). Siehe auch Kessler, SAK 18, 1991, 269 ff.

${ }^{41}$ Vollständige Übersetzung bei C. Husson, L'offrande du miroir dans les temples égyptiens de l'époque gréco-romaine, Lyon, 1977, 115.
} 
(16) D II, 149,9-12 Raum F, Ostwand, Nordseite, 2. Reg.

Titel: „Stoffopfer. (Zu sprechen:) Kleid der Renenutet ...? ... im Haus der Neith. Die, die herausgeht (prt $r$ b 3 ) in der Nacht des Kindes in seinem Nest, dem großen Fest des ganzen Landes ..."

(17) D II, 156,15-157,1 Raum F, Ostwand, Südseite, 1. Reg.; Titel: ḅnk $m 3^{c} t$

Isis: „Gesprochen von Isis, der Großen, der Gottesmutter, der Herrin von I3t-dit, zu Gast in Dendara, der mit den trefflichen Plänen des Fürsten der Weißen Krone ${ }^{42}$, der Großen des Himmels, der Mächtigen auf der Erde, die Befehle im Palast erteilt, die geboren wird in Dendara in der Nacht des Kindes in seinem Nest, dem großen Fest im ganzen 〈Land〉."

(18) D III, 2, 4-7 Raum I, südlicher Türpfosten

„Isis, die Große, die Gottesmutter, das Auge des Re, die Herrin des Himmels, die Gebieterin aller Götter, Königin der oberägyptischen Könige, Königin der unterägyptischen Könige, ohne daß es ihresgleichen gibt unter den Göttinnen, die geboren wird in Dendara in der Nacht des Kindes in seinem Nest, dem großen Fest des ganzen Landes; der Horizontische (3hty) geht am Himmel auf in der Dämmerung, nachdem sie ihre Mutter zur Welt gebracht hat. Das ist die Tochter des Geb, indem sie dauerhaft ist in ihrem Heiligtum. Alle Menschen sind versammelt, um sie zu sehen. Die Götter feiern ein Fest, die Göttinnen fangen an zu jubeln, die von Dendara sind herausgekommen, indem sie jubeln."

(19) D III, 55,5-6 Raum J, westlicher Türpfosten

„Isis, die Große, die Gottesmutter, das Auge des Re, die Herrin des Himmels, die Gebieterin aller Götter, deren Mutter sie zur Welt gebracht hat in I3t-dit am Tag der Nacht des Kindes in seinem Nest. Das ist die Einzigartige, die Gebieterin der Götter und Göttinnen, ohne daß ein Gott ihr gleichkommt; Sothis am Himmel, die Herrscherin der Sterne, die Befehle erteilt auf dem Erdkreis."

(20) D III, 79,9-11 Raum J, Südwand, Westseite, 1. Reg.; Titel: ḅnk $m 3^{c} t$

Isis: „Gesprochen von Isis, der Großen, der Gottesmutter, der Herrin von I3t-dit, zu Gast in Dendara, der Fürstin auf dem Erdkreis, die geboren wird in Dendara in der Nacht des Kindes in seinem Nest, der Herrscherin, mit zufriedenem Gesicht, der Maat, der Erhabenen, der Mächtigen, die an der Spitze des Palastes ist, der Prächtigen, die den Göttern und Göttinnen Befehle erteilt."

(21) D III, 98,11-3 Raum J, Nische, Südwand, Westseite, 2. Reg.; Titel: ḅnk $m 3^{\complement} t$ Isis: „Gesprochen von Isis, der Großen, der Gottesmutter, der Herrin von I3t-dit, zu Gast in Dendara, dem Auge des Re, der Herrin des Himmels, der Gebieterin aller Götter, der Herrin der Maat, die von der Maat lebt, sie ist zufrieden, wenn nach ihr (= Maat) gehandelt wird für ihren Ka, die geboren wird in Dendara in der Nacht des Kindes in seinem Nest, dem großen Fest des ganzen Landes, der einzigartigen Göttin, zu der Millionen jubeln, die Befehle erteilt, um das Strahlen zu veranlassen."

(22) D III, 170,9-11 Türsturz in Raum M, Westseite; Titel: dws nt $r$

\footnotetext{
${ }^{42}$ Zur Lesung sr ḅdt als Beiname des Osiris siehe Winter in CdE 39, 77, 1964, 41-3, vgl. aber P. Vernus, Athribis, BdE 74, Le Caire, 1978, 255, Anm. (e).
} 
Hathor: „Gesprochen von Hathor, die vorn ist in Dendara, der Rat im ganzen Land, der Vornehmen, der Mächtigen, [...], die die Götter und Göttinnen preisen wegen der Größe ihrer Macht, die oberägyptische Königin am Himmel, die unterägyptische Königin auf der Erde, der Herrscherin im Westen und Osten, der Vornehmen, der Mächtigen, die geboren wird in Dendara am Tag der Nacht [des Kindes in] seinem [Nest]“.

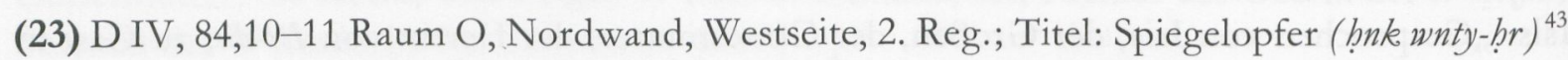
Isis: „... Gesprochen von Isis, der Großen, der Gottesmutter, der Herrin von I3t-dit, zu Gast in Dendara, mit schönem Antlitz, der Beliebten, die geboren wird in Dendara in der Nacht des Kindes in seinem Nest. Schu geht auf, nachdem sie die Augen geöffnet hat.“

(24) D IV, 104,10-3 Raum P, nördlicher Türpfosten

„Isis, die Große, die Gottesmutter, das Auge des Re, die Herrin des Himmels, die Gebieterin aller Götter, die den Leib ihrer Mutter Nut öffnet am Tag der Nacht des Kindes in [seinem] Nest. Sie beherrscht die beiden Länder, das Erbe Ägyptens, wenn sie das Amt schon in ihrer Windel ergreift. Tait, Herrin der $r$-Binde, die Uranfängliche, die Herrin der $s \check{s} d$-Binde ${ }^{44}$, für deren $\mathrm{Ka}$ das $n \underline{t} r y$-Kleid hergestellt wird, die Gebieterin, die Herrin des weißen Stoffes ( $\underline{b} \underline{d} t)$, die Einzigartige, die Herrin des grünen Stoffes (w $w d t$ ), die Fürstin des roten Stoffes (irtyw) ${ }^{45}$, die das Kleid prächtig macht, die Göttin, die an der Spitze der ḅwt-mnht (= Raum P) ist [...], wenn sie ihr Bruder in den Städten und Gauen schmückt, der seinen Sohn Horus auf den Thron setzt, ohne daß ein anderer es ihm gleichtun könnte."

(25) D IV, 124,14-5 Raum P, Nordwand, 2. Reg.; Titel: Opfer des nach Lotus duftenden Gewandes (ḅnk $3 h t h(t) s \check{s} n)^{46}$

Isis: „Gesprochen von Isis, der Großen, der Gottesmutter, der Herrin von I3t-dit, zu Gast in Dendara, der Fürstin, die die Nut geboren hat, die zur Welt gekommen ist in Dendara in der Nacht des Kindes in seinem Nest ..."

(26) D IV, 133,2-4 Raum P, Soubassement, Südwand „... Er (= der König) bringt dir (= Isis) die Flut ( $m$ tr) mit der Salbe in seiner Gestalt, indem du (?) erhaben bist [...] deinen Leib zu salben in der Nacht des Kindes in seinem Nest, dem Fest des Bekleidens der Herrin (?) [...], um die Neunheit in dem Heiligtum zu salben ..."

(27) D IV, 228, 4 Raum S, Außenmauer, Westseite

Isis: „Die Königin von Ober- und Unterägypten, die geboren wird in Dendara in der Nacht des Kindes in seinem Nest ...“"

(28) D IV, 230,6-7 Raum S, Innenmauer (Nordwand), Ostseite „Es bleibt der Ort der Freude des Re (st $\left.3 w t-i b n R^{e}\right)$ [am] Tag bei seinen schönen Festen an den Epagomenen ${ }^{47}$, (in) der Nacht des Kindes in seinem Nest."

\footnotetext{
${ }^{43}$ Vollständige Übersetzung bei Huss on, op. cit., 139-40.

${ }^{44}$ Siehe hierzu weiter unten Abschnitt V.

${ }^{45}$ Vgl. hierzu zuletzt R. Germer, Die Textilfärberei und die Verwendung gefärbter Textilien im alten Ägypten, ÄA 53, Wiesbaden, 1992, 129-30; weitere Belege bei Edel, ZÄS 102, 1975, 20-1.

${ }^{46}$ Zur Lesung vgl. D IX, 119,2. Für 3ht. „Kleid“ o. ä. vgl. das Determinativ $\gamma$ in D II, 224, ult; D III, 160,7; D IV, 124,9 (vgl. auch D III, 160,4 und D IX, 68,7).

${ }^{47} 5$ brw (w) bryw rnpt, die normale Schreibung für die Epagomenen, die genaue Tagesbezeichnung folgt in Ap-
} 
(29) D IV, 234,6-10 Raum S, obere Randinschrift, Westseite „... Er (= der König) hat das Heiligtum (hwyt) erbaut für Iwnt in Dendara und Harsomtus, der an ihrer Seite ist, um ihre Götterbilder zu schmücken bei ihrer Prozession mit weißem

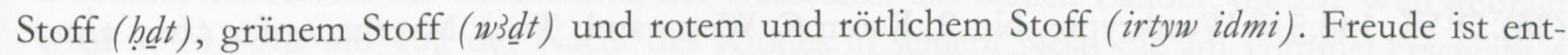
standen vor T3-n-Itm am IV. šmw 29, dem Fest des Empfangens der Kleidung für Re, den von H3-di und der ganzen Neunheit von Dendara. Sie erscheinen in ihrem Tempel, während der Horizontische strahlt. Sie erfüllen das Land mit Goldstaub. Die Götter feiern ein Fest, und die Göttinnen jubeln auf dem Fest des Empfangens der Kleidung für die Herrin von Dendara an diesem schönen Tag der Nacht des Kindes in seinem Nest, dem großen Fest des ganzen Landes. Sie möge viele Jahre geben ..."

(30) D V, 8,15-6 Ostkrypte Nr. 1, Raum B, Ostwand; Titel: zerstört Ptah: „Gesprochen von Ptah, der südlich 〈seiner〉 Mauer ist, dem großen Gott, zu Gast in Dendara, dem Erhabenen in seinem Wesen im $p r-R^{e}$, der die Nahrung deiner (fem.) Tochter zuweist in der st-mshnt am Tag der Nacht des Kindes in seinem Nest."

(31) D V, 135,11-2 Südkrypte, Raum B, Südwand; Titel: Eintreten zur Tür aus Gold Isis: „Gesprochen von Isis, der Großen, der Gottesmutter, der Herrin von I3t-dit, zu Gast in Dendara, $\underline{b} n m t-{ }^{c} n h$ in der st-mshnt, deren Gestalt geboren wurde in der Nacht des Kindes in seinem Nest, die die beiden Länder mit ihren Strahlen erhellt $(s\langle\underline{b}\rangle \underline{d})$. Gold: Höhe: 1 Elle.“

(32) D V, 142,8-9 Südkrypte Nr. 1, Raum C, obere Randinschrift, Südseite „Harsomtus, der lebendig Erglänzende am Himmel am Tag des Neujahrsfestes, der in seinen Tempel leuchtet in der Nacht des Kindes in seinem Nest, der die Strahlen zur Erde sendet in die Geburtsstätte. Der Himmel jubelt, die Erde freut sich, die Tempel der Götter sind glücklich, wenn er aufgeht in seinem Heiligtum in der $w \underline{t} s-n f r w$-Barke an seinem schönen Fest des tp-rnpt ..."

(33) D VI, 64,5-7 Südkrypte Nr. 4, Südwand; Titel: ḥnk $m 3^{\complement} t$

Isis: „Gesprochen von Isis, der Großen, der Gottesmutter, der Herrin von I3t-dit, zu Gast in Dendara, der Tochter des Geb, die die Nut geboren hat, die zur Welt gebracht wurde in $s t-R^{e 48}$ am Tag der Nacht des Kindes in seinem Nest. Ihr Vater geht auf am Himmel in der Nacht, während ihre Mutter in T3-rr gebiert.“

(34) D VI, 66,10-1 Westkrypte Nr. 1, Türöffnung, Nordseite

„Der König von Ober- und Unterägypten kommt zu dir, Isis, Große, Gottesmutter, Herrin von I3t-dit, zu Gast in Dendara, Auge [des Re] ...? ... ${ }^{49}$ Jubel herrscht auf dem Fest des Jahres am Tag der Nacht des Kindes [in seinem Nest ...].“

(35) D VI, 87,10-1 Westkrypte Nr. 1, Raum D, Ostwand; Titel: Spiegelopfer ${ }^{50}$ „... Ich komme zu dir, Fürstin des $p r_{-}^{e} n w t$ (= Dendara), Herrscherin von Ägypten. Ich hebe

position, vgl. z. B. M. Alliot, Le culte d'Horus à Edfou, BdE 20, Le Caire, 1949, 207, Zl. $18: 5$ hrww ḅryw rnpt mswt 3st (=4. Epagomenentag); Zl. 10:5 hrww hryw rnpt mswt Nbt-ḥwt (= 5. Epagomenentag), d. h. hier ist grh nhn $m$ sš.f Apposition zu 5 hrww hryw.rnpt, womit insgesamt die Nacht des 5. Epagomenentages gemeint ist.

${ }^{48}$ Nach Gauthier, DG V, 82 ein Heiligtum im Norden von Dendara.

49 nty?

${ }^{50}$ Vollständige Übersetzung bei Husson, op. cit., 149-50. 
hier die beiden Scheiben des Himmels hoch für Deine Majestät am Tag der Nacht des Kindes in seinem Nest."

(36) D VI, 148,5-7 Westkrypte, Nr. 2, Raum C, Westwand; Titel: Myrrhenopfer (šms 'ntyw) Isis: „Gesprochen von Isis, der Großen, der Gottesmutter, der Herrin von I3t-dit, zu Gast in Dendara, dem göttlichen Falkenweibchen ${ }^{51}$, der Herrscherin von Punt, der Fürstin in den beiden Ländern und auf der Erde, die geboren wird in Dendara in der Nacht des Kindes in seinem Nest. Es erstrahlt der Leuchtende, nachdem sie ihre Augen geöffnet hat.“

(37) D VI, 162,3-4 Westkrypte Nr. 3, Ostwand, 1. Reg.; Titel: ḅnk ms ${ }^{c} t$

Isis: „Gesprochen von Isis, der Großen, der Gottesmutter, der Herrin von I3t-dit, zu Gast in Dendara, der Fürstin, die die Nut geboren hat, die zur Welt gekommen ist in T3-n-Itm am Tag der Nacht des Kindes in seinem Nest ..."

(38) D VII, 125,12-4 Raum U, Ostwand, Südwand, Soubassement

Isis: „Gesprochen von Isis, der Großen, der Gottesmutter, der Herrin von I3t-dit, zu Gast in Dendara, dem Auge des Re, der Herrin des Himmels, der Gebieterin aller Götter, die zur Welt gebracht wird in T3-rr am Tag der Nacht des Kindes in seinem Nest, der Königin der Göttermächte $(s h m w)$, die das Überschwemmungswasser herausgießt aus dem Bein (sty), die das Feld mit den Ackerpflanzen ergrünen läßt ..."

(39) D VIII, 69,3 Kiosk auf dem Dach (W'), Tableau bei 11. Säule „Die gute Mesechenet ${ }^{52}$, die deinen Ka vollkommen macht wegen der Gebieterin an diesem schönen Tag der Nacht des Kindes in seinem Nest.“

(40) D IX, 49,15-7 Raum Z, Nordwand, Ostseite, 2. Reg.; Titel: Spiegelopfer ${ }^{53}$

Isis: „Gesprochen von Isis, der Großen, der Gottesmutter, der Herrin von I3t-dit, zu Gast in Dendara, mit freundlichem Gesicht, der Beliebten, die geboren wird in $\operatorname{Pr}-N f r-h r r$ (= Dendara) in der Nacht des Kindes in seinem Nest, dem großen Fest des ganzen Landes. Die beiden Himmelskörper gehen auf in der Dämmerung, wenn sie 〈ihre Augen〉 ${ }^{54}$ öffnet in T3-n-Itm. “

(41) D IX, 56,8-11 Raum Z, Ostwand, 3. Reg.; Titel: ḥnk ḥh

Amun: „... Den süßen Lufthauch Geben. Zu sprechen: Ich komme eilends nach T3-rr, dem Ort des Gebärens der sḩm-špst. Ich betrete die st-mshnt, indem ihre Türflügel geöffnet sind, um den schönen Nordwind an deine (= König) Nase zu geben ...? ... der süße Lufthauch ist in meiner Faust. Ich bringe (ihn) vor dich an diesem schönen (!) Tag des Kindes in seinem Nest.“

(42) D IX, 109,6-7 Raum Z, 6. Säule; Titel: Lotusopfer ${ }^{55}$

Isis: „Gesprochen von Isis, der Großen, der Gottesmutter, der Herrin der Götter in Dendara, die zur Welt gebracht wird in P $\underline{b} r$-t3.wy (=Dendara) am Tag der Nacht des Kindes in seinem Nest."

\footnotetext{
${ }^{51}$ Lesung bikt ntrt, vgl. D VI, 3,2 und 93,1-2.

52 mshnt nfrt, Beiname der Isis, Derchain-Urtel, LÄ IV, 107 s. v. Mesechenet.

${ }^{53}$ Vollständige Übersetzung bei Husson, op. cit., 167.

${ }^{54}$ Vgl. Inschrift Nr. (8).

${ }^{55}$ Vollständige Übersetzung bei M.-L. Ryhiner, L'offrande du lotus, Rites Égyptiens VI, Bruxelles, 1986, 86-7.
} 
(43) D IX, 183,9-11 Raum B', Ostwand, Südseite, Soubassement

Isis: „Gesprochen von Isis, der Großen, der Gottesmutter, der Herrin von I3t-dit, zu Gast in Dendara, der Herrin der Rechitleute, der Gebieterin der beiden Länder, die zur Welt gebracht wird in T3- $\mathrm{n}$-Itm am Tag der Nacht des Kindes in seinem Nest, die am Himmel erscheint an ihrem Anfang des Jahres, die die Überschwemmung aus den Quellöchern herbeiführt, die Herrscherin, die Herrin des Lebens, die an der Spitze der Götter ist, die das Leben für die Lebenden herbeiführt."

(44) D IX, 202,11-2 Südl. Türpfosten zu Raum $C^{\prime 56}$

„Dieser schöne Tag der Nacht des Kindes in seinem Nest, das große Fest im ganzen Land. Erscheinen der Hathor, der Herrin von Dendara und ihrer Neunheit in der Nacht vor diesem Tag. Umkreisen des Tempels. Durchführung aller Rituale. Niederlassen an ihren Orten.“ Vor diese Zeile gehören D IX, 203,12-204,6 (=Alliot, op. cit., 241, Zl. 23-5= Mariette, op. cit., I, 62 1). Am Ende: „Tag $5^{57}$ der Epagomenenen“, zu der dann brw pn nfr grḥ nhn $m$ š́.f Apposition wäre.

(45) D IX, 249,1-2 Passage $C^{\prime}-\mathrm{H}^{\prime}$, südl. Türpfosten

„Die Königin von Ober- und Unterägypten, das Mädchen, mit schönen Erscheinungen, die Beliebte bei den Göttinnen, die zur Welt gebracht wurde vor der st-mshnt am Tag der Nacht des Kindes in seinem Nest."

(46) J. Dümichen, Baugeschichte des Dendaratempels, Strassburg, 1877, Tafel XIX, ult.-XX, 1 Raum G', Südwand, Westseite, untere Randinschrift

„Hathor, die Herrin von Dendara, die vor I3t-dit leuchtet, der Stätte des Horus (?). Sie wird geboren von ihrer Mutter Nut [...] die Herrin von Dendara, die an der Spitze des pr-nsr ist, in deren Umkreis alle s3w.n.sn-Götter ${ }^{58}$ sind, die an der Spitze des Heiligtums ist, die mit leuchtenden Erscheinungen in ihrer Kapelle am Tag der Nacht des Kindes in seinem Nest.“

(47) Dümichen, op. cit., Tafel XLI-XLII = Cauville, BIFAO 90, 1990, 88 Raum G', untere Randinschrift an der westlichen Außenwand

„Der (weibliche) Horus-Re, die Vornehme, die geboren wird von Nut, die Herrin des Himmels, die geboren wird in Hwt-nbwt (= Dendara), die Herrin der Rechit, Hathor, die an der Spitze von Dendara ist, die Tochter des Re, die Erstgeborene des Atum, die schöne Frau, die am Uranfang zur Welt gebracht wurde, die Schai schon auf den Geburtsziegeln erhöht hat, für die Re-Behedeti Somtus aufgeht in der Dämmerung, nachdem sie geboren wurde am Tag der Nacht des Kindes in seinem Nest."

(48) LD, Text II, 219, 5. Säule, 1. Reihe (Raum G')

"Gesprochen von Isis, der Großen, der Gottesmutter, der Herrin von I3t-dit, zu Gast in Dendara, der Tochter der Nut in Hwt-...? ... (= Dendara $)^{59}$, die geboren wird in Dendara, um das ganze Land zu beherrschen am Tag der Nacht des Kindes in seinem Nest, der Herrscherin, der Gebieterin der Göttinnen, die die Jahre ergreift, die die Einkünfte einsammelt als diejenige, die

\footnotetext{
${ }^{56}$ Bei M. Alliot, Le culte d'Horus à Edfou, BdE 20, Le Caire, 1949 auf S. 242. Die Zeilen 25 und 26 stoßen nicht direkt aneinander, Mariette, Dend. I, $621+\mathrm{m}$ ist irreführend, vgl. D IX, 203-4 und 202 sowie Tafel 919.

${ }^{57}$ Nicht Tag 4, die Ergänzung von Alliot nach Brugsch (siehe die Einleitung) ist nicht begründet.

${ }^{58} \mathrm{Zu}$ dieser Göttergruppe siehe J.-C. Goyon, Les dieux gardiens, BdE 93, Le Caire, 1985, 449-96.

${ }^{59}$ Kopie vermutlich ungenau, vielleicht Hwt-Nwt. 
am Anfang entstanden ist, $\underline{b} n m t$, die den roten Stoff (ins) liebt, die Herrin der Beliebtheit, die Gebieterin der Götter und Göttinnen, die Befehle erteilt dem, der hinter ihr geht.“

(49) LD, Text II, 224 5. Säule, 2. Reihe (Raum G')

„Gesprochen von Isis, der Großen, der Gottesmutter, der Herrin von I3t-dit, zu Gast in Dendara, dem Auge des Re, der Herrin des Himmels, der Gebieterin aller Götter, der Tochter des Geb, die von der Nut geboren wird, die zur Welt gebracht wird in T3-rr, für die ihr Vater aufgeht, nachdem sie geboren wurde in der Nacht des Kindes in seinem Nest."

(50) Brugsch, Thes. 772 Pronaos $\left(G^{\prime}\right)$, Westwand (d. h. geographische Südwand) „... Re-Behedeti Somtus, er geht auf am Himmel in der Dämmerung am Tag der Nacht des Kindes in seinem Nest, dem großen Fest des ganzen Landes."

(51) Dümichen, op. cit., Tafel VII, paenult.-VIII, 2 (=4) Obere Randinschrift, südliche Außenwand

„Es entsteht das Falkenweibchen, $r p^{i} t t$, Tochter des $r p^{i} t$ (=Geb), Isis, die Große, die Gottesmutter, die erste Gemahlin des Onnophris, wahr an Stimme, die Mutter des Falken. Ihre Mutter hat sie zur Welt gebracht $(b \underline{b} r$ t3) in I3t-dit am Tag der Nacht des Kindes in seinem Nest. Daraufhin sagte ihre Mutter Nut über sie: ,Das ist Isis. Isis ist ihr Name in Wahrheit.' Die Vornehme in der Geburtsstätte. Re-Behedeti Somtus geht für sie auf in der Dämmerung, nachdem sie geboren wurde."

(52) Daumas, Mammisis de Dendara, 189,16-90,2 (auch Brugsch, Thes., 773,84) „Gesprochen von Isis, der Großen, der Gottesmutter, der Herrin von I3t-dit, zu Gast in Dendara, der Königin am Himmel, der Gebieterin der Sterne, der Herrscherin, der Herrin der Erbschaft, die an der Spitze des Palastes ist, der Ipet, der Großen, der mit vielen Jahren, die zur Welt gebracht wurde unter den Göttinnen, dem Falkenweibchen, der Erhabenen, der Mächtigen, der Fürstin, der Tochter des Fürsten, des Aufgehenden (wbnw), die geboren wird von der Nut, die an der Spitze ist, die am Anfang entstanden ist am Tag der Nacht des Kindes in seinem Nest. Re erstrahlt am Himmel in der Dämmerung, nachdem sie geboren wurde. Die Götter und Göttinnen preisen ihren Namen.“

(53) Brugsch, Thes. 96 Kleiner Isistempel, Ostwand (?) oder Westwand (?), so auf S. 1377 (die gleiche Inschrift)

„Das Falkenweibchen, die Jugendliche, die Tochter des Herrschers, Isis, die Große, die Gottesmutter, die empfangen wird in Dendara in der Nacht des Kindes in seinem Nest auf der Westseite des Hwt-š̃s $t^{60}$."

\section{Die Orientierung des großen Hathortempels und des kleinen Isistempels}

1. Wie an anderer Stelle gezeigt werden konnte, fand eine der Gründungszeremonien des großen Hathortempels in der Nacht vom 16. auf den 17. Juli -53 statt, genauer in den Morgenstunden des 17. Juli, die Querachse des Hathortempels und die Hauptachsen der beiden Geburtshäuser sind dabei auf den Aufgangsort der Sothis im Horizont hin ausge-

${ }^{60}$ Damit ist der kleine Isistempel gemeint, vgl. Text (58). 
richtet ${ }^{61}$. Das Azimut des Siriusaufgangs für den ebenen Horizont betrug zu dieser Zeit $72^{\circ} .4$ Süd über Ost nach Nord ${ }^{62}$. Da der heliakische Aufgang aber erst in über $2^{\circ}$ Höhe zu beobachten ist, wenn also der Stern schon weiter nach Süden gewandert ist, wurde seinerzeit der Vorschlag gemacht, daß die eigentliche Ausrichtung des Tempels schon einige Monate früher erfolgte, zu einer Zeit, als Sirius irgendwann in der Nacht und damit direkt im scheinbaren Horizont aufging ${ }^{63}$. Geben jetzt aber die obenstehenden Inschriften die Information, daß die Isis-Sothis in dem kleinen Tempel (I3t-dit) in der Nacht des Kindes in seinem Nest geboren wird, womit der heliakische Aufgang gemeint ist, und bedenkt man, daß die Achse des kleinen Tempels gegenüber der Querachse des großen den publizierten Plänen nach etwa um $3^{\circ}$ nach Süden abweicht ${ }^{64}$, so liegt die Schlußfolgerung nahe, daß auch der kleine Tempel eine astronomische Orientierung besitzt ${ }^{65}$. Die Querachse des großen Tempels besitzt ein Azimut von rund $72^{\circ} .5$ $\left( \pm 1^{\circ}\right)$ Süd über Ost nach Nord ${ }^{66}$, d. h. die Hauptachse des kleinen Tempels dürfte bei rund $69^{\circ} .5\left( \pm 1^{\circ}\right)$ liegen.

Die Rechenergebnisse ${ }^{67}$ lassen sich in einer Tabelle zusammenfügen:

\begin{tabular}{|c|l|c|c|}
\hline Zeit & \multicolumn{1}{|c|}{ Ereignis } & Höhe & Azimut \\
\hline $4^{\mathrm{h}} 17^{\mathrm{m}}$ & Siriusaufgang im Horizont & $0^{\circ}$ & $72^{\circ} .4 \mathrm{~S}$ über $\mathrm{O}$ \\
\hline $4^{\mathrm{h}} 30^{\mathrm{m}}$ & $\begin{array}{l}\text { heliakischer Aufgang des Sirius } \\
\text { (d. h. erstmalige Sichtbarkeit) }\end{array}$ & $2^{\circ} .3^{68}$ & $71^{\circ} .0$ \\
\hline $4^{\mathrm{h}} 45^{\mathrm{m}}$ & beste Sichtbarkeit $^{69}$ & $5^{\circ} .4$ & $69^{\circ} .2$ \\
\hline $5^{\mathrm{h}} 13^{\mathrm{m}}$ & Sonnenaufgang & $0^{\circ}$ & $115^{\circ} .2$ \\
\hline
\end{tabular}

D. h. die Achse des kleinen Tempels ist in etwa auf den Punkt des Horizonts hin ausgerichtet, an dem Sirius am Tag seines heliakischen Frühaufgangs am besten zu sehen ist.

2. Die genannte Bauinschrift enthält noch ein anderes Datum, nämlich den Tag, an dem Hathor in ihren Tempel eintrat ( $k$. $)^{70}$. Die beiden Herausgeber übersetzten das fragliche Datum mit „en l'an 1, le $19^{\mathrm{e}}$ jour du temps du dit roi (= Augustus)“, Devauchelle konnte jedoch zeigen,

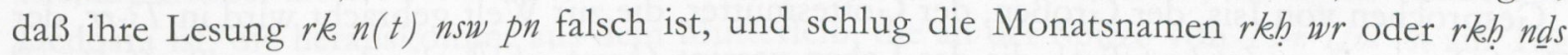

${ }^{61}$ Chr. Leitz, Studien zur ägyptischen Astronomie, ÄA 49, Wiesbaden, 58-9. Textgrundlage ist die von H. J. Amer und B. Morardet in ASAE 69, 1983, 255-8 publizierte Bauinschrift.

${ }^{62}$ So die Rechnung nach P. V. Neugebauer, Tafeln zur astronomischen Chronologie III, Leipzig, 1922 , 19.

${ }^{63}$ Vgl. hierzu auch P. V. Neugebauer, Astronomische Chronologie, Berlin/Leipzig, 1929, 144-5.

${ }^{64}$ Siehe die Pläne bei F. Daumas, Dendara et le temple d'Hathor, RAPH 29, Le Caire, 1969, pl. 1; G. Castel, F. Daumas und J.-C. Golvin, Les fontaines de la porte nord, Le Caire, 1984, plan 1.

${ }^{65}$ Diese Vermutung schon bei Wells in SAK 12, 1985, 290, der darauf jedoch nicht weiter eingeht.

${ }^{66}$ Leitz, op. cit., 58-9.

${ }^{67}$ Nach P. V. Neugebauer, Tafeln zur astronomischen Chronologie III, $\int 10$ (Zeit des Siriusaufgangs); $\int 12$ (Zeit des Sonnenaufgangs); \17 (Azimut und Höhe des Siriusaufgangs); \ 18 (Azimut des Sonnenaufgangs); $\int 19$ (Azimut des Siriusaufgangs für den ebenen Horizont). Alle Rechnungen bezogen auf Dendara, 17. Juli (jul.) -53. Das genaue Baudatum (unter Ptolemaios X?, siehe z. B. S. Cauville, Le temple de Dendara, Le Caire, 1990, 87) spielt hierbei keine Rolle, die Aufgangsorte (abhängig von der Deklination des Sterns) ändern sich nur sehr langsam.

${ }^{68}$ Dies der Mittelwert der in OLZ 30, 1927, 445-6 veröffentlichten Beobachtungen.

${ }^{69}$ Diese $28 \mathrm{~min}$. vor Sonnenaufgang sind der Mittelwert der drei in dieser Hinsicht verwertbaren Beobachtungen (d. h. Nr. 1, 2 und 4) des zuletzt genannten Aufsatzes.

${ }^{70}$ H. J. Amer und B. Morardet, ASAE 69, 1983, 256. 
(=II. oder III. prt) vor ${ }^{71}$, es sich mithin um den 14. Februar oder den 15. März im Jahre -28 handelt. U. U. läßt sich eine Entscheidung mit Hilfe der Astronomie fällen, hierzu muß aber noch einmal kurz die Schreibung des Datums betrachtet werden. Amer und Morardet lesen die Tageszahl gs $(=1 / 2) 1 / 101 / 30$, was als Summe 19/30, also den 19. Monatstag ergibt. Vergleicht man jedoch die ähnlich geschriebenen Daten in den Bauinschriften von Edfu, so stellt man fest, daß an den drei Stellen, wo gs: „1/2“ verwendet wird, gs jedesmal mit dem Ideogrammstrich geschrieben wird ${ }^{72}$, vermutlich, um Verwechslungen mit der Präposition $m$ auszuschließen. Im vorliegenden Text steht dagegen hinter dem fraglichen Zeichen kein Ideogrammstrich, sodaß vielleicht nur „am 4. (Monatstag) des rkh ...? ...“ zu lesen ist, d. h. die beiden in Frage kommenden Daten der 31. Januar bzw. der 29. Februar -28 wären. Das Aufgangsazimut der Sonne (= der Hathor) betrug für das erstere Datum $72^{\circ} .2$ von Süd über Ost nach Nord ${ }^{73}$, würde also fast zur Gänze mit dem Anfangsazimut der Sothis (oben $72^{\circ} .4$ ) und der Orientierung des Tempels übereinstimmen, wobei hinzuzufügen ist, daß sich zu dieser Jahreszeit die Deklination der Sonne und damit die Aufgangsazimute sehr schnell ändern, durchschnittlich alle 4-5 Tage um $1^{\circ 74}$. Dies Ergebnis sollte jedoch nicht überbetont werden, da das Datum nicht zweifelsfrei feststeht, vielleicht handelt es sich auch nur um einen Zufall ${ }^{75}$.

\section{Die Geburt der Isis-Sothis als dunkelrote Frau (st kmt ď̌rt)}

1. Die nachfolgenden Texte über die Geburt der Isis-Sothis als dunkelrote Frau ${ }^{76}$ sind von einiger Bedeutung für die Geschichte der Astronomie, da sie in einer noch genauer zu bestimmenden Form Bezug auf die rote Farbe des Sirius nehmen. Es folgt zunächst eine Übersetzung der bislang veröffentlichten 8 Texte $^{77}$ über die st kmt dšrt, die vor allem verdeutlichen soll, $\mathrm{da}$ diese Texte in genau den gleichen Zusammenhang wie die weiter oben angeführten Inschriften gehören, dann folgt eine Darstellung des Problems aus astronomiegeschichtlicher Sicht und zum Abschluß eine Neubewertung auf Grund des ägyptischen Materials.

(54) D I, 64,3-6 Sanktuar (A), Westwand, 2. Reg.; Titel: Stoffopfer (ḅnk mnht) "Gesprochen von Isis, der Großen, der Gottesmutter, die zur Welt gebracht wird in T3-rr, der dunkelroten Frau (st kmt ďstrt), die das Leben vereint $\left(\underline{b} n m t-^{-} n b\right)^{78}$, die den roten Stoff $(\text { ins })^{79}$

${ }^{71}$ In RdE 36, 1985, 173, Anm. 8.

${ }^{72}$ De Wit, CdE 36, 1961, 76, 290 und 293.

${ }^{73}$ Rechnung nach Neugebauer, op. cit., $\int 18$.

${ }^{74} \mathrm{Vgl}$. die Tafel 15 bei Neugebauer, op. cit.

${ }^{75}$ Genauso, wie es vielleicht Zufall sein wird, daß das Datum der Einweihung der hypostylen Halle aus dem 21. Jahr des Tiberius, am 21. Hathor (=17. Nov. 34, Devauchelle, RdE 36, 1985, 174; Übersetzung bei Daumas, op. cit., 31) fast das gleiche Aufgangsazimut aufweist, nämlich $71^{\circ} .4$ Süd über Ost nach Nord, aber eben nur fast das gleiche; der mit dem 31. Januar korrespondierende Tag liegt drei Tage früher (14. November, vgl. die Tafel 15 bei Neugebauer, op. cit.). Damit die Sonne erst bei $71^{\circ} .4$ den Tempel trifft, müßte im Osten schon eine kleine Mauer stehen.

${ }^{76}$ Zwei der Texte sind schon erwähnt bei F. Boll, Antike Beobachtungen farbiger Sterne, ABAW, Bd. 30,1. Abt., München, 1916, 15, der aber auf die Dendaratexte nicht weiter eingeht, seine Notiz ist in der Ägyptologie jedoch ohne Resonanz geblieben.

${ }^{77}$ Ein weiterer ist oben Text (9).

${ }^{78}$ Dies ist auch der Name der sechsten der sieben Kühe aus Tb. Spr. 148, deren Farbe rot war, wie aus ihrem Beinamen insyt hervorgeht, Tb (Naville), Bd. II, 378, Nr. 8 und Tb (Hornung), 299.

${ }^{79}$ Die Identifikation als „rot, blutrot“ ist sicher, vgl. außer den Wb-Einträgen (I, 100,4-13) Jéquier, BIFAO 
liebt, die Herrin der Beliebtheit, die Gebieterin der Göttinnen, deren Mutter sich freut, wenn sie sie sieht. Die Große ist groß in Ntry (= Dendara) als die mit großer Beliebtheit, die Gebieterin der Frauen beim Aufgang in Dendara, beim Bestrahlen der Erde, beim Erfreuen des Herzens der Neunheit, sie ist die göttliche Tochter, die herauskommt aus Geb, nachdem sie die beiden Länder schon in ihrer Windel geerbt hat."

(55) D I, 87,5-6 Couloir mystérieux, Außenseite des Sanktuars, Südwand, Westseite, untere Randinschrift

„I3t-dit in Dendara, wo sie geboren wird als dunkelrote Frau, $\underline{n} n m t-{ }^{\circ} n h$, die den ins-Stoff liebt, die Gebieterin der Göttinnen [...] ..."

(56) D VI, 2, ult. - 3,7 Südkrypte Nr. 2, Ostseite, obere Randinschrift

„Die Königin von Ober- und Unterägypten, das Auge des Re, die Gebieterin der beiden Länder, die Uräusschlange, die an der Spitze des Hwt-š̌st ist, die große mḅnt-Schlange auf dem Kopf des Atum, die das Herz des Re bei seinem Aufgang füllt, die Geschmückte, die Herrin des Schmucks, die Herrin des Palastes, $B \not h d t y t^{80}$, die buntgefiederte, das göttliche Falkenweibchen ${ }^{81}$, die Herrin von Punt, ...? ..., die das Kind beschützt bei seinem Erscheinen, B3t, die Herrin von Ntry, die an der Spitze des südlichen Bubastis (= Dendara) ist. Das ist Hatmehit, die Herrin von Mendes, die Schöne ( $n w t$ ), Iwnt, die Herrin von Dendara, die Fürstin auf dem Erdkreis, die Herrin der Göttermächte (shmw), für deren Majestät man das wtt aufstellte ${ }^{82}$, die dunkelrote Frau, $\underline{b n m t-}{ }^{n} h$, die den ins-Stoff liebt, ... sie ist es, die das Überschwemmungswasser aus seinem Quelloch herausholt.“

(57) D IX, 18,12-4 und 19,5-14 = Brugsch, Thes., 97 und 1379 Raum Z, Eingang, Türpfosten, Westseite

„Dendara ist hoch und dauerhaft, nachdem die Isis darin geboren wurde als dunkelrote Frau, $\underline{b n m t-} n h,[\ldots]$, die Herrin der Beliebtheit, die Gebieterin der Göttinnen, die Vornehme, die Schöne, die das Leuchten ( $w b n)$ des Sonnenlichtes ( $\check{s} w)$ sieht am Himmel in der Dämmerung, nachdem sie geboren wurde in dieser Stadt. ...

Isis, die Große, die Gottesmutter, die Goldene, die Herrin von Dendara, die an der Spitze von Dendara ist, die herauskommt aus dem Mutterleib, die an der Spitze von T3-n-Itm ist am Tag der Nacht des Kindes in seinem Nest. Re geht auf am Himmel in der Dämmerung, nachdem sie zur Welt gebracht wurde in T3-rr als dunkelrote Frau, mit schönem Gesicht, mit festlich geschmückten Augen $^{83}$, Herrin der Beliebtheit, Gebieterin der Göttinnen, die Große, schön Anzusehende ... [...] im Mutterleib [...], ohne daß es ihr Herauskommen gibt.

Der Himmel feiert ein Fest, die Erde jauchzt, Hwt-nhm (= Dendara) jubelt $(\mathrm{nhm})$ vor ihr, nachdem sie ( = die Bewohner von Dendara) die Herrscherin gesehen haben, die ihre Mutter in Dendara an dem großen Fest des ganzen Landes geboren hatte ...

Sothis am Himmel, die das Überschwemmungswasser aus seinem Quelloch herausholt, die das Ackerland begrünt zu seiner Zeit, die am Himmel wandert auf dem Wege ihres Bruders, die

19, 1922, 140-1; AEO I, 65-6 und vor allem Alliot, RdE 10, 1955, 1-7; zuletzt auch noch R. Germer, Die Textilfärberei und die Verwendung gefärbter Textilien im alten Ägypten, ÄA 53, Wiesbaden, 1992, 126-8.

${ }^{80} \mathrm{Vgl}$. Fairman in BIFAO 43, 1945, 100.

${ }^{81}$ Vgl. D VI, 93,1-2; 148,5.

${ }^{82}$ Diese Passage zitiert bei Ch. Sa mbin, L'offrande de la soi-disant „clepsydre“, Studia Aegyptiaca XI, Budapest, 1988, 226, zur Stelle selbst vgl. S. 42-3 (= Edfou IV, 82).

${ }^{83} \mathrm{Zu}$ ș̣b mndty vgl. Meeks, BIFAO 77, 1977, 81, Anm. 1. 
in seinem Schritt geht jeden $\mathrm{Tag}^{84}$, die Herrin des Himmels, die Gebieterin der Dekane (b3w $n h w)^{85}$, die die Jahre berechnet von ihrem Aufgang, B3t am Himmel, die Mächtige auf Erden ..."

(58) J. Dümichen, op. cit. (bei Text 46), Tafel VI, 1-3 untere ${ }^{86}$ Randinschrift auf der südlichen Außenwand

„Der (weibliche) Horus-Re, die Große, die geboren wird in Dendara auf der Westseite des Hwt-š̌š $t^{87}$. Behedeti geht auf am Himmel in der Dämmerung, nachdem sie geboren wurde am

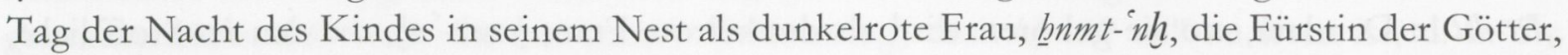
die Gebieterin der Zauberkraft, deren Mutter über sie sagt: „Ich bin wirklich deine Mutter“88. So entstand ihr Name Isis. Schai hat sie bereits auf ihren Geburtsziegeln erhöht, Thoth hat für sie die Titulatur festgesetzt ..."

(59) Dümichen, op. cit., Tafel XXXVII, 10-1 Raum G', obere Randinschrift, Westwand „Das ist Dendara, man sagt über diese Stadt: „Osiris, Horus, Isis“" ${ }^{\text {"D9 }}$. Das ist I3t-dit, das groß ist in seinem Namen, das ist das Geburtshaus ( $p r-m s$ ) der Nut, der Ort der Geburt ${ }^{90}$. Isis wird dort geboren als dunkelrote Frau, $\underline{b n} n t-{ }^{e} n h$, die den ins-Stoff liebt ... ${ }^{91}$.“

(60) Dümichen, op. cit., Tafel XXXVIII, 8-9= Brugsch, Thes., 1378. Westliche Außenwand „Das ist (die Kapelle) $W^{c} r$-h $h p r$ - ḩ3t, so sagt man über diesen Bezirk; I3t-dit sagt man darüber, die Geburtsstätte (st-mshnt) der Isis, das ist das Geburtshaus der Nut, indem 〈ihre $\rangle^{92}$ Mutter sie zur Welt bringt an dieser Stätte am Tag der Nacht des Kindes in seinem Nest als dunkel〈rote〉 Frau, $\underline{b} n m t^{-} n h$, Herrin der Beliebtheit ..."

(61) Daumas, Mammisis, $32=$ Brugsch, Thes., 102, identisch mit 1378. Auf einer Wand des kleinen Isistempels

„An diesem schönen Tag der Nacht des Kindes in seinem Nest, dem großen Fest der Weite der Erde, (an dem) Isis geboren wird im Innern von Dendera von der Ipet, der Großen im Hwt-Ipt als dunkelrote Frau, $\underline{b n m t-}{ }^{\circledR} n h$, der Beliebten ..." $" 93$

2. Eine auch für Laien gut verständliche Darstellung der Frage, warum ein für möglich gehaltener Farbwechsel des Sirius von rot im Altertum zu weiß in der Gegenwart für die Astro-

\footnotetext{
${ }^{84}$ Gemeint ist der eine gute Stunde vor ihr aufgehende Orion, genauer wahrscheinlich $\beta$ Orionis (siehe dazu Chr. Leitz, Studien zur ägyptischen Astronomie, ÄA 49, Wiesbaden, 1989, 106 Index s. v.), die Himmelsbahn der beiden Sterne (abhängig von der Deklination $\delta$ ) war etwa die gleiche, vgl. $\delta$ ( $\alpha$ Canis majoris) für das Jahr $0=-15^{\circ} .96$ und $\delta$ ( $\beta$ Orionis $)=-12^{\circ} .60$, im Jahr -1400 hatten sie exakt die gleiche Himmelsbahn $\left(\delta=-17^{\circ} .95\right.$ bzw. $\left.-17^{\circ} .96\right)$, alle Werte nach P. V. Neugebauer, Tafeln zur astronomischen Chronologie I, Leipzig, 1912, Tafel III.

${ }^{85}$ Vgl. z. B. die Inschrift Brugsch, Thes., 14 auf der Decke der großen Säulenhalle.

${ }^{86}$ Vgl. PM VI, 80, oben.

${ }^{87}$ Parallele in Text (53), gemeint ist der kleine Isistempel.

${ }^{88}$ is $n w r(. i)$ mwt.t; für inw, $n w$ als unabhängiges Personalpronomen der 1. Sing. vgl. H. Junker, Grammatik der Denderatexte, Leipzig, 1906, 42, \55 $\beta$.

${ }^{89}$ So schon Dümichen, ZÄS 7, 1869, 105, dem Fairman in ASAE 43, 1945, 251, Bemerkung VIII zustimmt.

90 st mshnt, vgl. Tafel XLI und Wb II, 148.

${ }^{91}$ Der Rest ähnlich wie Text 58.

${ }^{92}=$ Isis.

${ }^{93}$ Das Folgende ist wieder ähnlich wie bei den anderen Inschriften, eine vollständige Übersetzung bei D a u mas, op. cit.
} 
nomie von so großer Bedeutung ist, gibt der Astronom W. Schlosser in einem kürzlich erschienenen Artikel ${ }^{94}$.

„Der gegenwärtige Stand der astrophysikalischen Kenntnisse beruht zunächst auf dem Beobachtungsbefund, daß die große Mehrzahl der Fixsterne ihre Oberflächentemperatur und ihre Gesamtstrahlungsleistung so adjustiert, daß nur ausgewählte Kombinationen von diesen beiden Größen vorkommen (Hertzsprung-Russell-Diagramm). Dieses statische Bild wird durch Modellrechnungen (und entsprechende Rechnungen) gestützt, die für einen gerade entstandenen Fixstern eine solche feste Kombination zwingend vorschreiben, wobei die Gesamtmasse dieses Sterns entscheidend eingeht (sogenannte „Hauptreihe").

Mit der Umwandlung von Wasserstoff in Helium im Zentrum des Fixsterns tritt nun ein dynamisches Element in die Betrachtung ein. Wiederum abhängig von der Masse ändert der Stern seinen inneren Aufbau und paßt mit zunehmender Geschwindigkeit seine Oberflächentemperatur und Strahlungsleistung an. Im Falle der Sonne dauert dies etwa 10 Milliarden Jahre (von denen die Hälfte bereits um ist). Der Stern bläht sich auf und wird insgesamt heller, wobei aber seine Oberflächentemperatur absinkt. Aus dem Stern ist ein „Roter Riese“ geworden. Auch dieses Stadium währt nicht unbeschränkt. Der Stern steuert eine Endphase an, zum Beispiel das Stadium eines „Weißen Zwerges“. Ein Weißer Zwerg besitzt eine ziemlich hohe Oberflächentemperatur. Sein Durchmesser (etwa Erdgröße) ist aber so gering, daß er insgesamt sehr wenig Leistung abstrahlt, also recht lichtschwach ist.

Alle diese Entwicklungslinien basieren - zumal in ihren zeitlichen Abläufen - allein auf Modellrechnungen. Die diesen Rechnungen zugrundeliegenden Vorstellungen sind aber sicher in manchen Punkten zu einfach und korrekturbedürftig. Grundsätzlich sind alle derart abgeleiteten Zeiträume (etwa von der Entwicklung eines Roten Riesen zum Weißen Zwerg) so groß, daß eine merkliche Änderung in historischen Zeiten nicht zu erwarten ist. Damit ist jede Beobachtung eines Farb- bzw. Helligkeitswechsels von eminenter Bedeutung, auch für die theoretische Astrophysik, da sie deren Grundvorstellungen zu bestätigen oder zu widerlegen vermag.

Hierin liegt die Wichtigkeit einer von den bisher bekannten Nachrichten unabhängigen Quelle über die rote Farbe des Sirius. Sirius erscheint ja heute weiß, so daß dieser Stern, genauer: die Komponente Sirius B, einen Farbwechsel in kurzer Zeit durchgemacht haben muß.“

Da ein solcher Farbwechsel nach den heutigen astrophysikalischen Vorstellungen in so kurzer Zeit als ausgeschlossen gilt, wurden schon früh zwei Erklärungen erwogen ${ }^{95}$ :

(a) Bei Sternen in geringer Höhe über dem Horizont verändert die Farbszintillation nach zufälliger Verteilung deren Farbe, ein weißer Stern flackert gleich häufig rot, grün oder blau. Diese Erklärung ist nicht sehr überzeugend, da, wenn eine Verteilung auf mehrere Farben möglich ist, die Festlegung auf rot unerklärt bleibt.

(b) Bis $3^{\circ}$ Höhe über dem Horizont erreicht der Sirius die rote Farbe der Betelgeuze ( $\alpha$ Orionis) oder übertrifft sie, d. h. die antiken Quellen nennen den Sirius rot, weil er zur Zeit seines (heliakischen) Aufgangs rot erscheint.

Die ausführlichsten Angaben hierfür liefert Hephaistion von Theben, nach dem die älteren

\footnotetext{
${ }^{94}$ Bergmann/Schlosser in Francia, Forschungen zur Westeuropäischen Geschichte, 15, 1987, 43-4. Eine Kurzfassung mit stärkerer astronomischer Ausrichtung erschien in Nature, Vol. 318, Nov. 1985, 45-6, eine weitere Darstellung bei Mucke in: Der Sternenbote, Österreichische Astronomische Monatsschrift, Astronomisches Büro Wien, 34. Jahrgang, 419, 1991, 126-33. Ein lesenswerter wissenschaftsgeschichtlicher Überblick über das Problem, das seit den Zeiten von Thomas Barker (1760) bis heute immer wieder die Astronomie beschäftigt hat (unter anderen Sir John Herschel und Alexander von Humboldt), findet sich bei D. Malin/P. Murdin, Colours of the Stars, Cambridge, 1984, 88-91.

${ }^{95}$ Vgl. außer den genannten Artikeln auch Gundel in RE, 2. Reihe, III, 327 s. v. Sirius.
} 


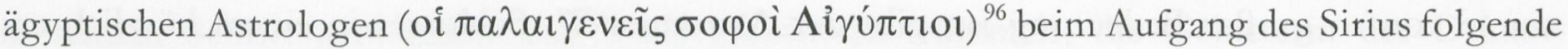
Färbungen des Sterns registriert und begutachtet haben ${ }^{97}$ :

1.) $\chi \rho \cup \sigma o \varepsilon \imath \delta \dot{s}$ [like gold].

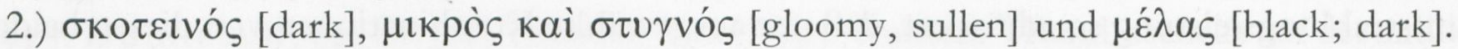

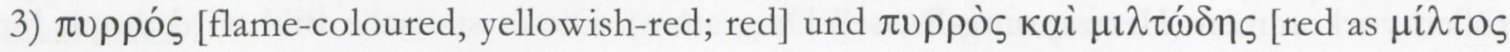
(= red earth, red ochre, ruddle)].

4.) $\chi \lambda \omega \rho$ ós [greenish-yellow, pale green; yellow] und $\mu$ «

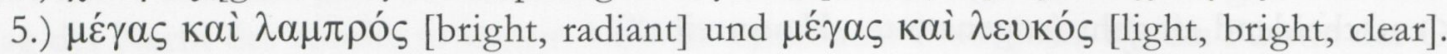

Die Frage ist also, ob sich die roten Farbbezeichnungen des Sirius im Altertum wie beispielsweise bei Claudius Ptolemaios ${ }^{98}$ oder bei Seneca ${ }^{99}$ hauptsächlich von der Farbe seines Aufgangs herleiten oder ob tatsächlich der Stern einen Farbwechsel durchgemacht hat.

3. Das ägyptische Material vermag hierzu nun von zwei Seiten zu einer Klärung beizutragen.

a) Wer akzeptiert, daß die Texte (9) und (54)-(61) die Geburt der Isis-Sothis behandeln, wofür $\alpha$ ) die Nennung des genauen Datums ${ }^{100}$ und $\beta$ ) die Erwähnung der Sothis selbst ${ }^{101}$ spricht, der hat automatisch über das Datum die Information, daß es um eine Beobachtung des Sirius bei seinem heliakischen Aufgang, also in geringer Höhe über dem Horizont geht, in der er in der Tat rot sein kann. Die gängige Übersetzung als schwarzrote Frau ${ }^{102}$ trifft dabei den tatsächlichen Sachverhalt nur ungenau, da deutsche (englische, französische) Farbwörter nicht ohne weiteres mit den ägyptischen Farbbezeichnungen gleichgesetzt werden können. Macht man sich die Ergebnisse von Schenkel zueigen ${ }^{103}$, so bezeichnet $\mathrm{km}$ etwa „dunkel“, der Gegensatz ist $\underline{b d}$ : „hell“, $d \check{s} r$ : die „warmen Farben“, ws $\underline{d}$ : die „kalten Farben“; für $d \check{s} r$ schlägt er folgende deutsche Entsprechungen vor: „rot, orange, gelb, (hell)braun, rosa (violett, lila)“. Würde man nach

${ }^{96}$ Vgl. hierzu den demotischen Papyrus Cairo 31222, Hughes in: JNES 10, 1951, 256-64, dessen erhaltene Reste einen sehr ähnlichen Aufbau wie das in der folgenden Anm. zitierte Hephaistionkapitel aufweisen. Vgl. hierzu auch pGen. inv. 90 bis (Sijpersteijn/Wehrli, Museum Helveticum 42, 1985, 328-33). Zu den Begutachtungen des Siriusaufgangs siehe auch die Inschrift des Harchebis, Derchain, CdE 64, 1989, 77-8.

${ }^{97}$ Apotelesmatika I, 23, zitiert nach der Zusammenfassung von Gundel, op. cit., 327, dessen Zitate nach der neueren Ausgabe von D. Pingree, Hephaistio Thebanus Apotelesmatica, Bd. I, Leipzig, 1973, 66-73 überprüft wurden; die Angaben in [eckigen Klammern] übersetzen die Farbbezeichnungen nach H. G. Liddell/R. Scott, A Greek-English Lexikon, 9. Auflage, Oxford, 1940.

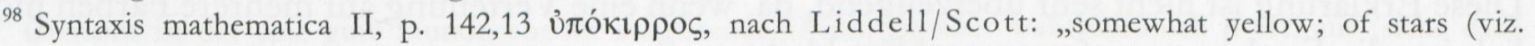
those now called red) mit Bezug auf Ptolemaios, Tetrabiblos 23,90 (dort als Farbe des Aldebaran ( $\alpha$ Tauri), der ein eindeutig roter Stern ist), vgl. hierzu F. Boll, Antike Beobachtungen farbiger Sterne, ABAW, Bd. 30, München, 1916, 52.

${ }^{99}$ Quaest. Natur. I, 17, zitiert von Boll in: Neue Jahrbücher für das Klassische Altertum, Bd. 39, 1917, 26. „Er (= Seneca) bemerkt hier, es sei nicht zu verwundern, daß die Ausdünstungen der Erde verschieden seien, da ja auch am Himmel nicht eine und dieselbe Farbe erscheine (non unus appareat color rerum), sondern der Hundsstern stark, der Mars mäßig rot sei, der Jupiter aber gar keine rote Farbe zeige, vielmehr einen rein weißen Glanz (acrior sit Caniculae rubor, Martis remissior, Jovis nullus in lucem puram nitore perducto).

${ }^{100}$ Nämlich die Nacht des Kindes in seinem Nest = der 5. Epagomenentag in Text (9), (57), (58), (60) und (61). Dieses Datum schließt damit den einzigen noch prinzipiell in Frage kommenden Himmelskörper, die Sonne, aus, deren Geburt regelmäßig auf den I. 3ht 1 gelegt wird, vgl. z. B. Kitchen, Ram. Inscr. VI, 564,8 und meine kommende Arbeit über die Tagewählkalender am entsprechenden Datum.

101 Text (57).

102 Z. B. Daumas, Mammisis, 33.

${ }^{103}$ In ZÄS 88, 1963, 131-47. 
diesem Schema die genannten Farbbezeichnungen des Hephaistion für die Siriusaufgänge auf die ägyptischen Termini verteilen, so erhielte man in etwa folgendes Bild:

\begin{tabular}{|c|c|}
\hline $\mathrm{km}$ & 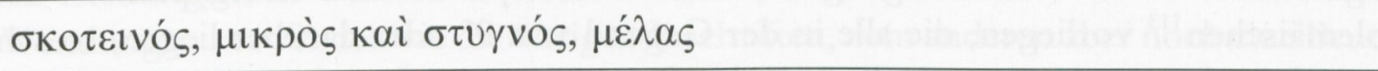 \\
\hline$d \check{s} r$ & 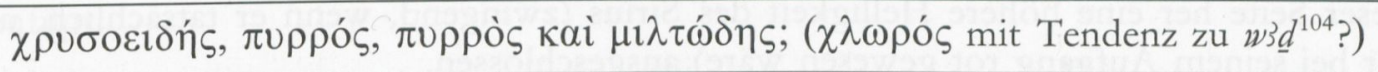 \\
\hline$\underline{b \underline{d}}$ & 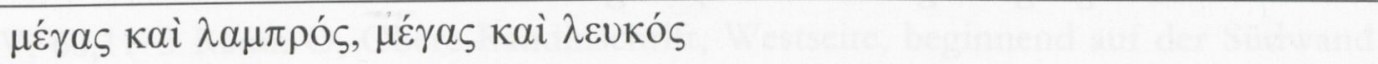 \\
\hline
\end{tabular}

D. h. eine Bezeichnung der aufgehenden Isis-Sothis als st kmt dšrt würde ziemlich genau mit den natürlichen Verhältnissen übereinstimmen, ansonsten ist nur noch für einen anderen Himmelskörper eine ägyptische Farbbezeichnung überliefert, nämlich für den Mars, den die Ägypter den roten Horus ( $H r d \breve{s} r$ ) nannten, dessen Rotfärbung der Sirius bis zu einer Höhe von $3^{\circ}$ noch übertrifft ${ }^{105}$.

b) Wenn Sirius bzw. genauer die Komponente Sirius B im Altertum ein Roter Riese und nicht wie heute ein Weißer Zwerg war, so muß die Gesamthelligkeit wesentlich größer gewesen sein, die Berechnungen ${ }^{106}$ ergaben eine Gesamthelligkeit von rund $-4.0 \mathrm{~m}$ (vergleichbar der Venus) gegenüber heute $-1.5 \mathrm{~m}$, d. h. Sirius wäre im Altertum zehnmal so hell gewesen wie heute $^{107}$. Eine solche Schlußfolgerung, astrophysikalisch zwingend, hat aber Konsequenzen für den Sehungsbogen (arcus visionis).

Der Sehungsbogen eines Sterns ist die für die Sichtbarkeit des Sterns erforderliche Mindesthöhe der Sonne unter dem Horizont, er ist folgendermaßen definiert ${ }^{108}$ : Der Sehungsbogen $\beta$ ist die Höhe der Sonne unter dem wahren (nicht für Refraktion korrigierten) Horizont in dem Augenblick, in dem der Stern in diesem wahren Horizont steht. Er ist von drei Faktoren abhängig:

(1) Der Größe des Sterns; je heller ein Stern ist, umso kleiner ist sein Sehungsbogen.

(2) Von der Azimutdifferenz (Lage des Aufgangsortes) des Sterns und der Sonne; je größer die Differenz im Azimut, desto kleiner der Sehungsbogen.

(3) Von den Witterungsverhältnissen ${ }^{109}$.

Faktor (2) und (3) werden von der obigen Annahme nicht getroffen, wohl aber Faktor (1). Hätte Sirius in etwa die gleiche Helligkeit wie Venus gehabt, so hätte er auch in etwa deren Sehungsbogen, d. h. $6^{\circ} .2$ oder $6^{\circ} .3^{110}$. Ein Sehungsbogen von dieser Größe würde aber ge-

\footnotetext{
${ }^{104}$ Vgl. W. Till, Farbenbezeichnungen im Koptischen in: Studia Biblica et Orientalia III (Anal. Bibl. 12, 1959) 341 оүшт als Wiedergabe von $\chi \lambda \omega \rho o ́ \varsigma$ (mir nicht zugänglich, zitiert nach Goyon in: Livre du centenaire, MIFAO 104, Le Caire, 1980, 32; wohl Crum, CD, 493a).

${ }^{105}$ Vgl. die Angaben von Schlosser in Nature, Vol. 318, Nov. 1985, 46: Mars: 1.4 nach dem Farbindex B-V; Betelgeuze (die der Sirius übertreffen kann): 1.9, alle Sterne mit B-V > 1.0 galten im Altertum als „rot“.

${ }^{106}$ Schlosser, a. a. O.

${ }^{107}$ Die Differenz der Helligkeit zwischen zwei aufeinanderfolgenden Größenklassen beträgt $10^{0.4}=2.512$, d. h. im vorliegenden Fall 2.5.512 $=10$.

${ }^{108}$ Dies und das Folgende nach P. V. Neugebauer, Astronomische Chronologie, Berlin/Leipzig, $1929,153$.

${ }^{109}$ Hierin könnte ein weiterer Grund für den verhältnismäßig großen Sehnungsbogen von $\beta=11^{\circ}$ bei der Tempelgründung von Dendara liegen (siehe oben Abschnitt III). Das Gründungsdatum -53, 17. Juli (jul.) entspricht dem 15. Juli (greg.) und liegt somit etwa 25 Tage nach der Sommersonnenwende. Etwa zu dieser Zeit (16.-17. Juli greg.) war der Nil den Durchschnittswerten zufolge auf der Breite von Dendara schon so weit gestiegen, daß er über die Ufer trat [D. Bon nea u, La crue du Nil, Paris, 1964, 23], was zur Folge haben mußte, daß die Luftfeuchtigkeit und damit der Dunst am Horizont ebenfalls gestiegen war; ein Umstand, der in den entsprechenden Jahren die Sichtbarkeitsverhältnisse des Sirius natürlich verschlechterte.

${ }^{110}$ Vgl. die Tafel bei Neugebauer, op. cit., E 64 und die frühere in Tafeln zur astronomischen Chronologie III, Tafel 28 a.
} 
genüber dem sonst angenommenen von rund $9^{\circ} \mathrm{zu}$ einem drei, z. T. auch vier Tage früher zu beobachtenden heliakischen Aufgang des Sirius führen ${ }^{111}$. Da nun aber genügend Beobachtungen seines heliakischen Aufgangs sowohl aus dem pharaonischen Ägypten ${ }^{112}$ wie aus dem ptolemäischen ${ }^{113}$ vorliegen, die alle in der Gegend von $9^{\circ}$ oder darüber liegen, so scheint von dieser Seite her eine höhere Helligkeit des Sirius (zwingend, wenn er tatsächlich, und nicht nur bei seinem Aufgang rot gewesen wäre) ausgeschlossen.

\section{Das Herbeibringen der $s \check{s} d$-Binde}

Da die Geburt der Isis-Sothis in der Nacht des Kindes in seinem Nest unmittelbar vor Beginn des Neujahrs erfolgt, wird bisweilen auch das Ritual des Herbeibringens der $s \check{s} d$-Binde ${ }^{114}$ mit dem hrw grḥ nhn $m$ ss.f in Verbindung gebracht. Es handelt sich dabei um einen Stoffstreifen auf dem „Ein schönes Jahr millionen- und hunderttausendfach“ (rnpt nfrt ḥ̣ hfnw) steht ${ }^{115}$, den der König der Göttin überreicht. El-Kordy ${ }^{116}$ sieht in šsd ein aktives Partizip eines Kausativstammes zu $\check{s} d$ : „herausholen, herausziehen“ (z. B. den Nil), sodaß š̌d die Bedeutung hätte: „(der Stoff), der das Herausholen veranlaßt“", was dann je nach Kontext auf die Sothis, den Nil oder die Sonne am Neujahrstag gedeutet wurde. Ein Text war weiter oben schon genannt worden (Nr. 12), die beiden anderen werden im folgenden übersetzt; auch sie schließen wieder durch die gleichzeitige Nennung von $r n p t n f r t$ und grh $n h n n m$ šs.f eine Identifikation des fraglichen Tages mit dem 4. Epagomenentag aus.

(62) LD II, Text, 209= Brugsch, Thes. 103 Raum G', Westwand, 3. Reg.

Titel: „Herbeibringen der š̌d-Binde des schönen Jahres für Isis, die Große, die Gottesmutter. ,Empfange, empfange die schönen Jahre am Tag der Nacht des Kindes in seinem Nest!““

Isis: „Gesprochen von Isis, der Großen, der Gottesmutter, der Herrin von I3t-dit, zu Gast in Dendara, dem Auge des Re, Der Herrin des Himmels, der Gebieterin aller Götter, der Goldenen, die geboren wird in pr-nbwt, die zur Welt gebracht wird von ihrer Mutter Nut am Tag der Nacht des Kindes in seinem Nest ..."

(63) Daumas, Mammisis de Dendara, 192,3-5

Titel ${ }^{117}$ : „Herbeibringen der š́d-Binde des schönen Jahres. Bereiten des Weihrauchs für das schöne Jahr."

Isis: „Gesprochen von Isis, der Großen, der Gottesmutter, der Herrin von I3t-dit, zu Gast in Dendara, der Tochter der Nut in Hwt-Nwt (Dendara), die geboren wird in Dendara, die ihren Vater schützt am Tag der Nacht des Kindes in seinem Nest, der Hekat, der Herrscherin der Göttinnen, die das Jahr ergreift ..."

${ }^{111}$ Rechnung nach Neugebauer, Astronomische Chronologie, Tafel E 61.

${ }^{112}$ Vgl. die Zusammenfassung bei Leitz, op. cit., 91. Eine Herabsetzung des Sehungsbogens bei den dort genannten Fällen (Tagewählkalender, Eberskalender, Senenmut und Nutbild) ist nicht möglich, da der Siriusaufgang jedesmal in einem zeitlich genau festgelegten Abstand zu einem anderen astronomischen Ereignis steht. Vgl. auch das letzte Kapitel weiter oben über die Orientierung und Gründung des Dendaratempels.

${ }^{113}$ Bei Ptolemaios gar $11^{\circ} .7$, so Borchardt und Neugebauer in OLZ 30, 1927, 447.

${ }^{114}$ Die Farbe dieser Binde wird nicht genannt. Die Angabe aus Pyr. 1147a, daß sie von roter Farbe (t $\left.\underline{t} m s t\right)$ sei, wird man kaum auf die Ptolemäerzeit übertragen dürfen.

${ }^{115}$ Vgl. die Abbildung in LD II, Text, 209.

${ }^{116}$ In Fs Gutbub, 127. Vgl. zu dem Ritual auch Goy on in: Hommages à François Daumas, Montpellier, 1986, 331-2.

${ }^{117}$ Eine vollständige Übersetzung bei El-Kordy in Fs Gutbub, 128-9. 


\section{Die Inschrift D IV, 60,11-3 - eine genaue Zeitangabe des Siriusaufgangs?}

Was den nachfolgenden Text so interessant macht, ist die Tatsache, daß er einerseits die gleichen Wendungen verwendet wie die übrigen Inschriften, zum anderen aber noch eine Bruchzahl hinzufügt.

(64) D IV, 60,11-3 Raum O, Obere Randinschrift, Westseite, beginnend auf der Südwand „Die Königin von Ober- und Unterägypten, Isis, die Große, die Gottesmutter, die Herrin von I3t-dit, zu Gast in Dendara, die geboren wird in Dendara $m 5$ ḩryw rnpt 1/2 1/10 1/3.f. Er geht auf $^{118}$, (nämlich) der Aufgehende am Himmel in der Dämmerung nach dem Öffnen ihrer Augen in T3-n-Itm. Die Götter feiern ein Fest, die Göttinnen, sie jubeln beim Sehen der Herrscherin $\left\langle\right.$ der Götter ${ }^{119}$ in der Nacht des Kindes in seinem Nest ..."

$\mathrm{Daß}$ es hier wieder um die Geburt der Isis geht, ist klar, schwieriger ist aber die korrekte Übersetzung des in Umschrift wiedergegebenen Datums. Der erste Teil $m 5$ hryw rnpt weist nicht die sonst übliche Schreibung auf, es fehlt $\operatorname{brw}(w)$. Theoretisch könnte 5 eine Kardinaloder eine Ordinalzahl sein, da ersteres aber keinen Sinn ergibt (Isis wird nur an einem der fünf Epagomenentage geboren und nicht an allen fünf), scheint mir eine Übersetzung: „am 5. der außerhalb des Jahres $=$ am 5. Epagomenentag“ richtig zu sein, zumal kurz danach noch wieder die Nacht des Kindes in seinem Nest erwähnt wird. Für den zweiten Teil gilt, daß nebeneinanderstehende Stammbrüche normalerweise zu addieren sind, merkwürdig ist hier die Reihenfolge, da in der Regel die kleinen auf die großen Brüche folgen ${ }^{120}$, Ausnahmen sind eher selten ${ }^{121}$. Ein Beispiel für die Verbindung einer Summe aus Stammbrüchen und einem Suffixpronomen fand sich weder bei Sethe noch in einer der gängigen Grammatiken, es scheint mir aber wahrscheinlicher, das Suffix auf die ganze Summe als nur auf das letzte Summenglied zu beziehen. D. h., der zweite Teil wäre zu übersetzen mit „in seinem $1 / 2+1 / 10+1 / 3=14 / 15^{\prime \prime}$.

Was damit gemeint ist, darüber kann nur der Gesamtkontext Auskunft geben. Thema ist wie in den anderen Inschriften die Geburt der Isis-Sothis, ihr heliakischer Aufgang. Einmal angenommen, das Suffix $f$ bezöge sich nicht auf den ganzen 5. Epagomenentag, sondern nur auf dessen Nacht, eben die Nacht des Kindes in seinem Nest, so läßt sich folgende Rechnung anstellen ${ }^{122}$ :

Sonnenuntergang (am 16. Juli) war um $18.81 \mathrm{~h}=18^{\mathrm{h}} 50^{\mathrm{m}}$, Sonnenaufgang (am 17. Juli) um $5.21 \mathrm{~h}=5^{\mathrm{h}} 13^{\mathrm{m} 123}$.

D. h. die Nachtlänge betrug 10.40 h, 14/15 davon sind 9.71 h, d. h. der fragliche Zeitpunkt der Geburt der Isis wäre $18.81 \mathrm{~h}+9.71 \mathrm{~h}=4.52 \mathrm{~h}=4^{\mathrm{h}} 31^{\mathrm{m}}$. Diese Uhrzeit entspricht genau der Zeit des heliakischen Aufgangs ${ }^{124}$. Die Höhe des Sirius betrug in diesem Augenblick $2^{\circ} .5$, das Azimut war $70^{\circ} .9$ Süd über Ost nach West ${ }^{125}$, was gut zu den Angaben in Abschnitt IV paßt, nach denen die Farbe des Sirius $(=s t k m t d s r t)$ bis zu einer Höhe von $3^{\circ}$ die rote Färbung der Betelgeuze erreichen oder übertreffen kann.

\footnotetext{
${ }^{118}$ Lies iw. $f$ wbn.

${ }^{119}$ Eine Ergänzung zu ḥnwt $\langle n \underline{t} r>w$ scheint mir wahrscheinlicher als der Plural von hnnwt.

${ }^{120}$ K. Sethe, Von Zahlen und Zahlworten bei den alten Ägyptern, Straßburg, 1916, 60.

${ }^{121}$ Westendorf, Med. Gramm., 115 und Tagewählkalender pCairo 86637, XXVIII, 6 (= IV. prt 8).

${ }^{122}$ Der Einfachkeit halber wieder für den 17. Juli (jul.) -53 wie oben.

${ }^{123}$ Rechnung nach P. V. Neugebauer, Tafeln zur astronomischen Chronologie III, \ 12.

${ }^{124}$ Siehe weiter oben die Tabelle in Abschnitt III.

${ }^{125}$ Rechnung nach Neugebauer, op. cit., \ 17.
} 
Um noch einmal auf den Text zurückzukommen: Es kann nicht klar nachgewiesen werden, daß sich das Suffix . $f$ tatsächlich nur auf die Nacht bezieht, und auch sonst ist die Lesung nicht völlig zweifelsfrei. Es scheint mir aber auf Grund des Gesamtkontextes [Geburt der Isis-Sothis] und der hohen Übereinstimmung zwischen der Rechnung unter den obigen Annahmen und den tatsächlichen Verhältnissen durchaus wahrscheinlich zu sein, daß eben diese Annahmen und damit die gegebene Textinterpretation richtig sind.

\section{Die Darstellung auf der Westwand des Schatzhauses (pr-ḥd; st nfrt $=$ Raum Q)}

1. Die folgende Stelle auf der Rückwand des $p r-\underline{h} \underline{d}$ wird deswegen gesondert angeführt, weil sie in einer Beziehung zu den Dekanen steht. Um dies besser verstehen zu können, ist eine Abbildung beigefügt, die die Lage der übersetzten Textpassagen markiert. Es handelt sich wie so oft um ein Maatopfer, diesmal nicht vor Isis, sondern vor Hathor ${ }^{126}$, im darüberstehenden 2. Register befindet sich in dem ganzen Raum eine Dekanreihe ${ }^{127}$, der Übergang vom alten zum neuen Jahr steht dabei genau über der Darstellung mit der Erwähnung der Nacht des Kindes in seinem Nest.

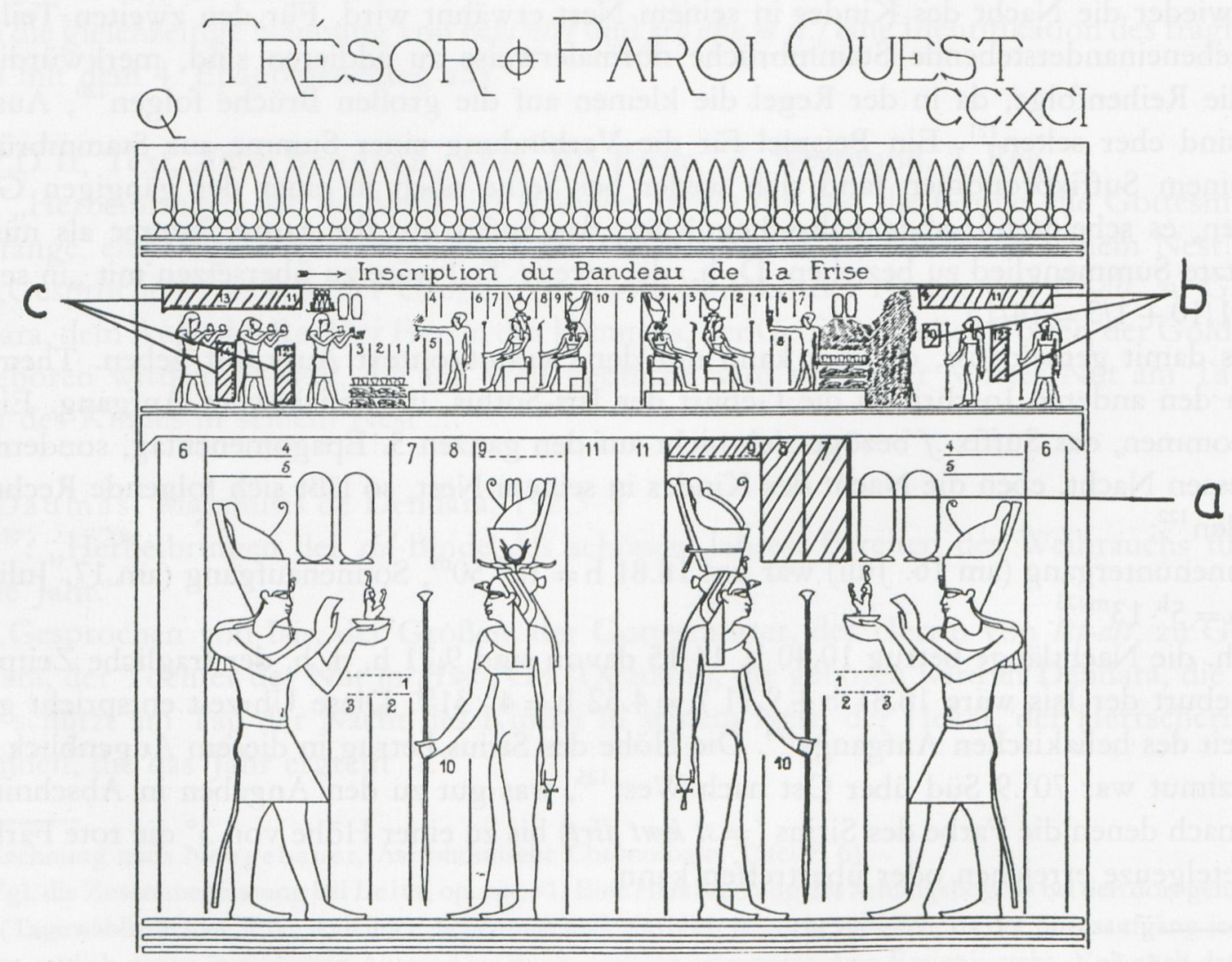

Abb. 2

\footnotetext{
${ }^{126}$ Vgl. Text (22) und (46).

${ }^{127}$ Eine vollständige Übersetzung bei Neugebauer-Parker, Astronomical Texts III, 134-40 und S. Aufrère, L'univers minéral dans la pensée égyptienne, BdE 105, Le Caire, 1991, 179-80.
} 
(65) D IV, 175,4-5 (=a)

"'Gesprochen von Hathor, der Großen, Herrin von Dendara, dem Auge des Re, der Herrin der Rechit, ${ }^{8}$ der Gebieterin der beiden Länder, die zur Welt gebracht wird vor 13t-dit am Tag der Nacht "des Kindes in seinem Nest."

D IV, 176,4 (= b)

„9Sothis. ${ }^{10}$ Gold. ${ }^{11}$ Onuris-...? ... ${ }^{128}$. ${ }^{12}$ Lapislazuli und Gold.“

D IV, 162 ult. - 163,1 (=c)

"11Snn. ${ }^{12}$ Quarz (mnw) und Gold. ${ }^{13} n h-m$-hrryw (Der von den Feinden lebt) ${ }^{14}$ Quarz (mnw) und Gold."

2. a) Bei dieser Reihe von Sterngöttern handelt es sich um eine Gruppe von insgesamt 59 Gottheiten ${ }^{129}$. Diese bestehen aus den 36 Dekanen und 12 weiteren Gottheiten, von denen jeweils einer drei Dekaden zugeteilt ist. Von den restlichen 11 sind der 2., 4., 6., 8. und 10. mit den fünf Kindern der Nut identisch (Osiris, Horus, Seth, Isis, Nephthys). Nach Parker ${ }^{130}$ handelt es sich dabei um eine Darstellung eines sog. dualen Jahres, einer Verbindung zwischen Mondjahr und bürgerlichem Jahr. Die jeweils vier Götter pro Monat (drei Dekane und ein „Zusatzdekan" ${ }^{\text {"131) }}$ ) seien dabei auf die vier Viertel eines Mondmonats aufzuteilen, was insgesamt 48 Gottheiten für ein normales Mondjahr von 354 Tagen ergebe. Die restlichen 11 Gottheiten entsprächen genau der Differenz der 354 Tage eines Mondjahres und den 365 Tagen des bürgerlichen Jahres, seien also mit diesen 11 Epagomenentagen gleichzusetzen.

b) $\mathrm{Zu}$ dieser Theorie ist folgendes zu sagen:

(1) Es handelt sich nicht immer um 59 Gottheiten, zumeist sind es weniger (so in Dendara), bisweilen sind es mehr ${ }^{132}$.

(2) Wenn es sich bei den 11 Gottheiten tatsächlich um 11 Epagomenengötter handelt, die den Unterschied zwischen einem Mondjahr und dem Sonnenjahr repräsentieren sollten, so wäre deren kalendarische Zuordnung jedenfalls festgelegt. Der 1. entspräche dem Jahrestag 355, der 2. dem Tag 356 usw. bis zum 11., der dem Tag 365 gleichzusetzen wäre. Genau das ist aber nicht der Fall. Die fünf Kinder der Nut, deren Geburt kalendarisch an die fünf Epagomenen (=Tag 361-365) gebunden ist, treten auf als Nr. 2, 4, 6, 8 und 10, so daß nach Ansicht des Verfassers die einzig erlaubte Schlußfolgerung ist, daß die restlichen 11 Gottheiten mit den fünf Epagomenentagen zu verbinden sind. Dabei gibt es theoretisch zwei Möglichkeiten:

a) Es gehören zusammen die Paare Nr. 1 und 2, 3 und 4 usw., isoliert bleibt Nr. 11 oder

$\beta)$ es gehören die Paare Nr. 2 und 3, 4 und 5 usw. zusammen, isoliert bleibt Nr. 1.

Diese Frage kann auf Anhieb nicht entschieden werden, wahrscheinlicher ist aber Möglichkeit $\alpha$ ), da der Name von Nr. 1 (fast) identisch ist mit Nr. 3 und 5, d. h. in dieselbe Gruppe gehören dürfte und Nr. 11 ( $(S n)$ als einziger auch sonst noch eine Rolle spielt, dazu weiter unten.

$\mathrm{Zu}$ dieser Interpretation, d. h. Bezug der 11 Götter nur auf die fünf Epagomenen, paßt auch,

\footnotetext{
${ }^{128}$ Lesung unsicher, Neugebauer-Parker, op. cit., 135: m3t t33y.

${ }^{129}$ Die ausführlichste Materialsammlung ist Kákosy in Oikumene 3, 1982, 163-91. Wichtig ist vor allem auch J. Vercoutter, Les objects égyptiens et égyptisants du mobilier funéraire carthaginois, Paris, 1945, 317-37.

${ }^{130}$ Calendars, 54-6, insbesondere S. 56. Die Interpretation wurde übernommen von Neugebauer-Parker, op. cit., 139 und E. Cruz-Uribe, Hibis Temple Project I, San Antonio 1988, 185-90.

${ }^{131}$ In der Liste bei Neugebauer-Parker, op. cit., 134-9 jeweils mit a gekennzeichnet.

${ }^{132}$ So bei Cruz-Uribe, a. a. O. Die Auslassung des Seth und der dazugehörenden Gottheit in einer Reihe von Dokumenten ist dagegen nichts Ungewöhnliches, sondern liegt im Rahmen ägyptischer Tradition (vgl. z. B. M. Alliot, Le culte d'Horus à Edfou, BdE 20, Le Caire, 1949, 207 und 214-5 [Auslassung des 3. Epagomenentages $=$ des Geburtstages des Seth]), d. h., dies widerspricht nicht seiner Theorie.
} 
daß beispielsweise im vorliegenden Text nicht alle 11 genannt werden, sondern nur die Nr. $1^{133}$, 3, 7, 9 und 11, also die zum 1. und 2. sowie 4. und 5. Epagomenentage gehörenden Gottheiten sowie die Nr. $11^{134}$, die Tatsache an sich, daß es für einen Jahrestag mehrere eponyme Gottheiten gibt, ist nichts Ungewöhnliches ${ }^{135}$.

3. a) Um auf die Darstellung zurückzukommen: Es konnte festgestellt werden, daß es sich bei ' $n h$-m-hryw um eine eponyme Gottheit des 5. Epagomenentages handelt, vor ihm steht der zwei $n w$-Krüge opfernde Snn, der nach einem Relief in Edfu in Verbindung mit dem neuen Jahr und der Überschwemmung steht ${ }^{136}$, das Opfer ist für „Isis, die Große, die Gottesmutter, die Herrin von I3t-dit", die angerufen wird als „Sothis, die Herrscherin über die Sterne, die den $b 3 w$ des Himmels folgt “ ${ }^{137}$. Insgesamt bezieht sich somit auch das ganze 2. Register auf die Nacht des 5. Epagomenentages, sowohl durch die Nennung von 'nh-m-hryw und Snn wie auch der Sothis selbst (in Text $65 \mathrm{~b}$ ), so daß der kalendarische Zusammenhang mit dem 1. Register, wo es um die Nacht des Kindes in seinem Nest geht, als sicher erscheint.

b) Bemerkenswert ist auch die Anordnung auf der Südwand, die den Durchgang zu den Räumen R und S, also zur „Chapelle du Nouvel An“ und der w bt ermöglicht, deren Verbindungen zum Neujahrsfest bekannt sind ${ }^{138}$. Außer den bereits genannten eponymen Gottheiten der Epagomenentage befinden sich auch noch die Dekane 28-36 in dem oberen Register der Südwand ${ }^{139}$. Nach dem pCalsberg I ist das Leben der Dekane in vier Abschnitte unterteilt ${ }^{140}$ :

\begin{tabular}{|r|l|}
\hline I & 80 Tage Aufenthalt im Osten des Himmels. \\
\hline II & $\begin{array}{l}120 \text { Tage Arbeit in der Mitte des Himmels (d. h. Kulmination nacheinander in der 12., } \\
11 ., \text { usw. bis zur 1. Nachtstunde). }\end{array}$ \\
\hline III & 90 Tage im Westen. \\
\hline IV & 70 Tage in der Unterwelt. \\
\hline
\end{tabular}

Auf einen bestimmten Tag bezogen heißt das, daß 8 Dekane im Osten stehen, 12 in der Himmelsmitte (=im Süden) kulminieren, 9 im Westen stehen und 7 in der Unterwelt verbleiben. D. h. die Nacht des Kindes in seinem Nest ist gerade der Übergang zwischen zwei Stufen. Die Tage davor galt, daß Nr. 28 die 12. Nachtstunde anzeigte, 29-36 im Osten standen, 1-7 in der Unterwelt waren. In dieser Nacht springt dann die Zählung um: Nr 1 (= Sothis) kommt

${ }^{133}$ Es handelt sich um D IV, 163, V (=19-20). Daß hierbei die Nr. 1 und nicht die hier namensgleiche Nr. 3 gemeint ist, folgt aus der Ikonographie. Nr. 1 ist eine liegende Schlange auf einem Sockel, Nr. 3 und 5 sind aufgerichtete Schlangen, vgl. CG 38924 (S. 234 der Publikation).

${ }^{134}$ Hierin liegt ein weiteres Argument für die Richtigkeit der obigen Annahme $\alpha$ ). Gesetzt den Fall, richtig wäre die Annahme $\beta$ ) und isoliert wäre die Nr. 1, so würde die im Text ausgelassene Nr. 5 mit dem 2. Epagomenentag (= Geburtstag des Horus) in Verbindung stehen, für dessen Wegfall besteht jedoch im Gegensatz zum 3. Epagomenentag (= Geburtstag des Seth) kein Grund.

${ }^{135}$ Man beachte, daß bis heute allein vier verschiedene Chronokratenlisten bekannt geworden sind, siehe Leitz, op. cit., 17-9.

${ }^{136}$ Siehe Kákosy in MDAIK 27, 1981, 255-7 mit Bezug auf Edfou I², 525 und 533 (Edfou XII, pl. 366-7).

${ }^{137}$ D IV, 162,8 und 10. Der Titel ist das Besänftigen der Sachmet (ș̣tp Shmt), deren unheilvolle Rolle an den Epagomenen gut bekannt ist (z. B. pLeiden I 348).

${ }^{138}$ Siche z. B. Daumas in LÄ IV, 468 s. v. Neujahr.

139 Zählung nach der Liste von Neugebauer-Parker, op. cit., 134-9.

${ }^{140}$ Das Folgende nach der Übersicht bei H. O. Lange und O. Neugebauer, Papyrus Carlsberg No. I, S. 60-1. Es handelt sich dabei um schematische Angaben, die für Rechnungen im Regelfall nicht verwendbar sind. 
aus der Unterwelt heraus, Nr. 28 wird danach die 11. Nachtstunde anzeigen. D. h. der erste in dieser Dekanreihe ist der, der die Grenze zwischen 12. und 11. Nachtstunde anzeigt, ungefähr zu diesem Zeitpunkt dürften sich auch die Priester im Raum $\mathrm{R}$ versammelt haben, um von dort über die Treppe (X) aufs Dach zu ziehen, um den Sothisaufgang zu beobachten.

\section{Die Neujahrsbezeichnung „Horn des Steinbocks“( $b$ ni3w)}

1. Die letzte Inschrift bringt die Nacht des Kindes in seinem Nest in einen Zusammenhang mit dem „Horn des Steinbocks“, einer nicht allzu seltenen Bezeichnung für das Neujahr in Edfu und Dendara ${ }^{141}$.

(66) D VIII, 98,9-11 (=Zl. 233-7 auf Tafel DCCCI), die Beischrift zum ersten herabsteigenden Priester der Treppe (X), der einen Naos mit folgender Beischrift trägt (D VIII, 99, ult. = Zl. 260-1):

„Isis, die Große, die Gottesmutter, die Herrin von I3t-dit, die den Himmel (k.bhow) durchwandert, die zum pr-wit zieht, Isis, die Große, die Gottesmutter."

Über dem Priester: „Der Sänger (iby) der großen Priester von I3t-dit, der das Götterbild sieht vor dem pr-wpr (= Raum J), der die Gottesmutter sieht auf ihrem erhabenen Fest am Tag der Nacht des Kindes in seinem Nest, der das Geheimnis ( $\check{s} t 3 w)$ vor die st-mshnnt (= Raum E) zieht am Tag des Festes des Re am Neujahrsfest (( $b b)$ wpt-rnpt), ebenfalls der Tag des Horns des Steinbocks ( $b$-nizw), millionenfach ohne Aufhören."

Interessant ist in diesem Zusammenhang auch der letzte Teil der oberen Randinschrift (D VIII, 87,2-3): „Hathor, ... die den König liebt. Sie möge ihm die Jahre der Sothis am Himmel geben ..."

2. Die Dokumente für die Verbindung des Steinbocks zum Neujahr hat Keimer gesammelt ${ }^{142}$, er konnte wahrscheinlich machen, daß ab dem NR die Hieroglyphe des Steinbocks oder auch nur sein Horn rnpt: „Jahr“" gelesen werden kann; als Grund nahm er die auffallende Ähnlichkeit des Steinbockhorns mit der Palmrispe (rnpt) an, das Wörterbuch (I, 173, 16) bietet die gleiche Erklärung für ${ }^{c} b$ nisw. Diese Interpretation scheint mir für die Lesung der Steinbockhieroglyphe überzeugend zu sein, für die Bezeichnung ${ }^{`} b$ nisw als Neujahr in ptolemäischer und römischer Zeit ist aber auch noch eine astronomische Erklärung denkbar. Das Horn des Zodiakalgestirns

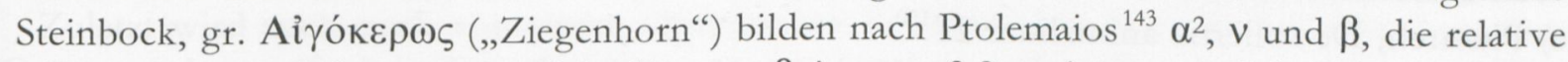
Helligkeit von $\alpha^{2}$ beträgt $m=3.8$, die von $\beta$ ist $m=3.2, v$ ist wesentlich lichtschwächer. Die Verbindung mit dem Neujahr könnte dadurch gegeben sein, daß die Sterne des Horns des Steinbocks kurze Zeit vor dem Siriusaufgang untergehen. In Zahlen sieht dies folgendermaßen aus:

Der Untergang von $\alpha^{2}$ Capricorni erfolgt am 17. Juli -53 um $4^{\mathrm{h}} 25^{\mathrm{m}}$, der von $\beta$ Capricorni schon um $4^{\mathrm{h}} 22^{\mathrm{m} 144}$, der heliakische Aufgang des Sirius war etwa um $4^{\mathrm{h}} 30^{\mathrm{m}}$ (siehe den Abschnitt III).

${ }^{141}$ Z. B. D II, 114, ult.; VII, 199,3; 201,2; IX, 48,10; Edfou I², 569,10; 583,11; III, 212,2; IV, 279,9 ; VI, 18,8. Der Wörterbucheintrag (I, 173,16 als „Bez. des Jahres“) dürfte dahingehend zu präzisieren sein, vgl. den nachfolgend übersetzten Text und den ganzen Abschnitt VIII.

${ }^{142}$ Intreprétation de quelques passages d'Horapollon, CASAE 5, Le Caire, 1947, 1-15.

${ }^{143}$ I. E. Heiberg, Ptolemaeus, syntaxis mathematica, Bd. II, Leipzig, 1903, 116-7, deutsche Übersetzung von K. Manitius, Ptolemäus, Handbuch der Astronomie, Bd. 2, Leipzig, 1913, 51. $\alpha^{2}$ ist dabei „von den drei im nachfolgenden Horn der nördliche“, $v$,der mittlere“ und $\beta$, der südliche der drei“.

${ }^{144}$ Rechnung nach P. V. Neugebauer, Tafeln zur astronomischen Chronologie III, \ 10. 
Diese Zahlen gelten freilich für den ebenen Horizont, in Wirklichkeit werden Sterne dieser Helligkeitsstufe schon einige Zeit früher infolge der Lichtschwächung in Horizontnähe (Extinktion) unsichtbar. Aus diesem Grund seien noch zwei weitere Zeitangaben gemacht. Um $4^{\mathrm{h}} 06^{\mathrm{m}}$ betrug die Höhe von $\alpha^{2}$ Capricorni noch $3^{\circ} .3$, die von $\beta 2^{\circ} .5$, um $4^{\mathrm{h}} 12^{\mathrm{m}}$ stand $\alpha^{2}$ nur noch $2^{\circ}$ über dem Horizont, dürfte also nur noch schwer sichtbar gewesen sein, während $\beta$ bei einer Höhe von nur noch $1^{\circ} .2$ sicher nicht mehr zu sehen war ${ }^{145}$.

3. Der Sachverhalt ist jedoch noch komplizierter, da eine Gleichsetzung des ägyptischen Steinbocks nisw mit dem Sternbild Steinbock (Capricornus) erst einmal fraglich ist, die demotischen Texte nennen es immer $h r_{-}{ }^{-} n h$ : „Ziegengesicht" ${ }^{\text {"146 }}$, wohl sicher nach dem griechischen

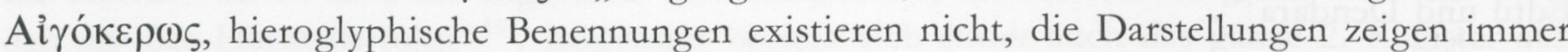
einen Ziegenkopf ${ }^{147}$. Die obige Interpretation wäre also recht fraglich, gäbe es nicht zum Glück noch einige Berichte klassischer Autoren, die leicht verändert den gleichen Sachverhalt beschreiben und den Weg in die richtige Richtung weisen.

a) Aelian, De natura animalium, VII, $8^{148}$

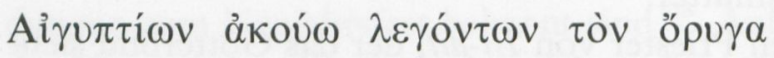

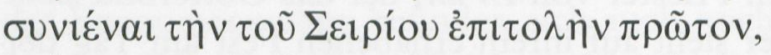

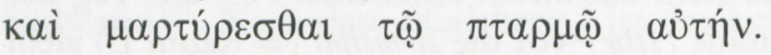

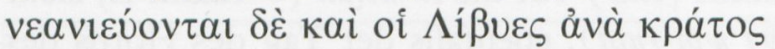

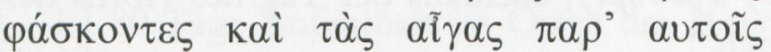

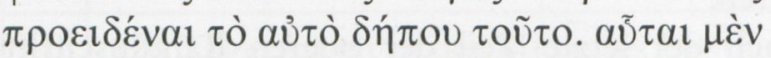

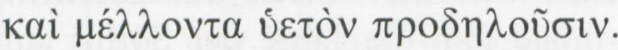

b) Plinius, Naturalis historia II, $107^{149}$

orygem appellat Aegyptus feram quam in exortu eius contra stare et contueri tradit ac velut adorare cum sternuerit.

Weit ausführlicher ist

c) Plutarch, De sollertia animalium $21^{150}$

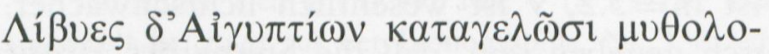

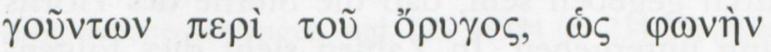

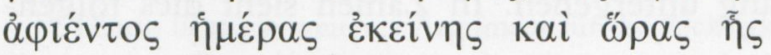

„Ich habe gehört, daß die Ägypter sagen, daß die Oryxantilope als erste den Aufgang des Sirius wahrnimmt und daß sie ihn durch ein Niesen bezeugt. Die Libyer prahlen gleichfalls, indem sie nach Kräften behaupten, daß auch die Ziegen bei ihnen eben dasselbe voraussehen. Sie künden nämlich ebenfalls klar den kommenden Regen an.“

„Ein wildes Tier in Ägypten, Oryx genannt, soll sich ihm [= Sirius], wie man sagt, bei seinem Aufgang entgegenstellen, ihn betrachten und durch Niesen gleichsam verehren."

„Die Libyer lachen über die Ägypter, die eine Geschichte über den Oryx erzählen, (nämlich) daß er einen Schrei ausstoße an jenem Tag und

\footnotetext{
${ }^{145}$ Alle Rechnungen nach Neugebauer, op. cit., \ 17.

${ }^{146}$ Z. B. Parker/Zauzich, in: Studies presented to Hans Jakob Polotsky, Massachusetts, 1981, 475; Neugebauer-Parker, Astronomical Texts III, 220, davor schon Spiegelberg, ZÄS 48, 1910, 148-9.

${ }^{147}$ Neugebauer-Parker, op. cit., 211.

148 Zitiert nach A. F. Scholfield, The Loeb Classical Library 448, London, 1971, 104-5 (= Hopfner, Fontes, 416-7), sehr ähnlich ist Damascius (= Hopfner, 690).

149 Zitiert nach C. Plinius Secundus d. Ä., Naturkunde Lateinisch-Deutsch, Buch II, Hrgb. R. König, HeimeranVerlag, 1974, 91 (= Hopfner, Fontes, 189).

${ }^{150}$ Zitiert nach Plutarch's Moralia, Bd. XII, Hrgb. H. Cherniss und W. C. Helmbold (The Loeb Classical Library 406), London, 1968, 410-1 (= Hopfner, Fontes, 265).
} 


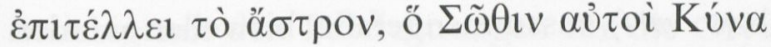

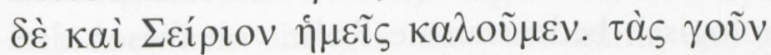

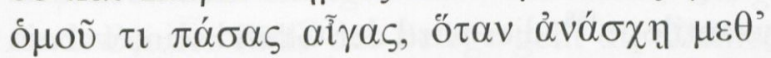

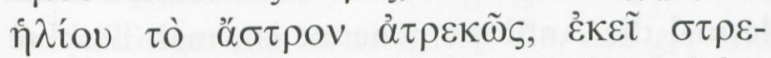

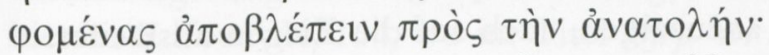

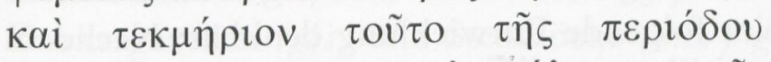

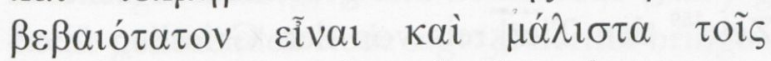

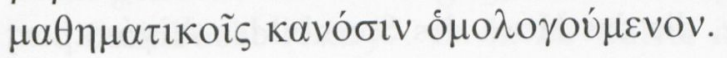

in (jener) Stunde, wenn der Stern aufgeht, den sie Sothis nennen, den wir aber Hundsstern oder Sirius nennen. Jedenfalls nun, wenn der Stern zusammen mit der Sonne aufgeht, dann drehen sich dort in der Tat fast alle Ziegen um und blicken nach Osten. Und dies ist der sicherste Beweis der Wiederkehr und stimmt aufs Beste mit den mathematischen Rechentafeln überein."

d) Ein Scholion zu Aratus [152] $]^{151}$

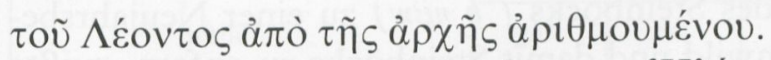

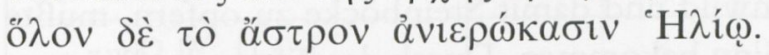

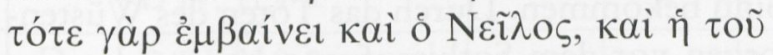

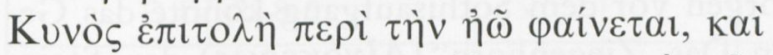

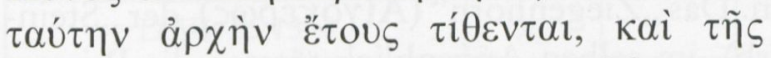

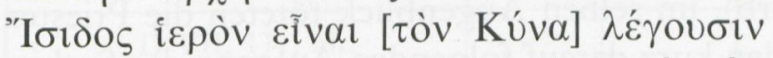

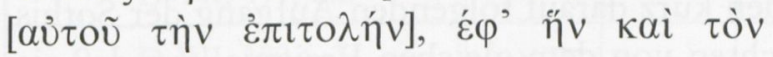

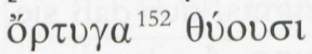

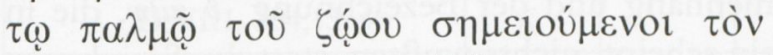

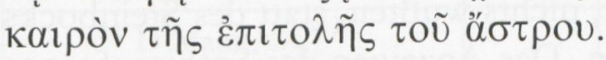

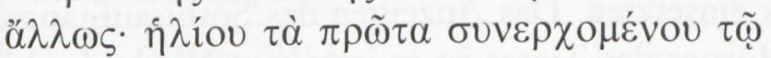
$\Lambda \varepsilon ́ o v \tau \imath$

„Von dem Anfang wird der Löwe gerechnet. Kurz nun, sie weihen den Stern der Sonne. Dann nämlich steigt auch der Nil, und der Aufgang des Hundssterns zeigt sich in der Morgendämmerung, und diesen machen sie zum Anfang des Jahres, und sie sagen, daß der Hundsstern der Isis heilig sei. Was nun seinen Aufgang betrifft, bei dem sie einen Oryx opfern, so zeigen sie durch das Zucken des Tieres den Augenblick des Aufgangs des Sterns an. Anders gesagt: Wenn die Sonne zum ersten Mal dem Löwen nahekommt.“

Faßt man den Inhalt der vier Texte zusammen, so stimmen sie alle darin überein, daß es sich um eine ägyptische Tradition über den Sothisaufgang handelt, zwei von ihnen präzisieren sogar, daß damit der Frühaufgang gemeint sei [c; d]. Alle nennen das Tier Oryx, wobei man wissen muß, daß Oryx bei den Griechen nur eine Sammelbezeichnung für verschiedene Antilopenarten war $^{153}$. Zwei Texte $[a ; c]$ geben aber auch die Information, daß sich die Ziegen ( $\alpha \tilde{i} \gamma \varepsilon \varsigma)$ gleich verhalten, sie stehen der Sothis gegenüber (d. h. im Westen) und blicken nach Osten [b; c]. Die Anzeige des Aufgangs erfolgt durch Niesen [a; b] oder Schreien [c], die Exaktheit dieser Ankündigung wird sogar mit den mathematischen Rechentafeln verglichen [c]. Zuletzt wird auch noch von einem Opferbrauch berichtet, bei dem die Ägypter einen Oryx töteten, dessen Zucken dann den Siriusaufgang anzeigen sollte [d].

Dieses Antilopenopfer am Neujahrstag läßt sich auch in Edfu und Dendara nachweisen, geopfert werden von den hier interessierenden Tierarten Dorkasgazelle (Gazella dorcas) = äg. $g h s=t 3 w^{154}$, Oryxantilope (Oryx gazella) $=\ddot{a} g . m 3-\underline{h} \underline{d}$ mit der Kurzform $m \underline{h}=h n p-w d \underline{d} s t=w d{ }^{155}$ und Steinbock $($ Capra ibex nubiana $)=\ddot{g}$. ni3 $w^{156}$. All diese Tiere wurden von den Ägyptern unter

${ }^{151}$ Zitiert nach J. Martin, Scholia in Aratum vetera, Stuttgart, 1974, 155. Ich bedanke mich bei Françoise Labrique, die mir bei der Übersetzung der Passage geholfen hat.

${ }^{152}$ Die Wachtel, lies: ő $\rho\{\tau\} \cup \gamma \alpha$ : „den Oryx“; dies auch die Ansicht von Hopfner, Tierkult, 99 und Gundel in RE, 2. Reihe, Bd. III, 335 s. v. Sirius.

${ }^{153}$ Siehe Steier in RE, 36. Halbband, 1530-1 s. v. Oryx.

${ }^{154}$ Für diese Identifikation siehe den Anhang.

${ }^{155}$ Eine seltene Bezeichnung für die Oryxantilope, nicht im Wb, vgl. aber Edfou VI, 256,6 und 257,12.

${ }^{156}$ Siehe die vier Belege in Edfu $=$ Edfou I², 537,11; 549,5; 553,15-6 und 555,9, eine Übersetzung der Gesamttexte bei M. Alliot, Le culte d'Horus à Edfou auf den Seiten 309-10, 380-1, 386-8 und 423-4. Die entsprechenden 
der Bezeichnung „Wild der Wüste“ ( $w$ t nt ḩsst bzw. mrt) zusammengefaßt, bisweilen werden auch noch die Ziegen dazugezählt ${ }^{157}$. Die mythologisch bedeutsamste Rolle von diesen drei Wüstentieren besaß in Ägypten zweifellos die Oryxantilope ${ }^{158}$, dies wird der Grund sein, warum in den griechischen und lateinischen Texten jedesmal die Antilope genannt ist, man beachte aber, daß Plutarch die Fähigkeit, das neue Jahr anzuzeigen, auch auf die Ziegen ausweitet.

Für die Entstehung des Begriffes ` $b$ nisw scheint folgende Entwicklung denkbar: Nachdem die Ägypter irgendwann in frühhellenistischer Zeit ${ }^{159}$ den Tierkreis von den Griechen übernommen hatten, stellte jemand fest, daß die Sterne, die das Horn des Sternbildes Aỉ $\gamma o ́ \kappa \varepsilon \rho \omega \varsigma$ bildeten, kurz vor dem Aufgang des Sirius untergingen. Da er gleichzeitig wußte, daß der Kopf des Steinbocks (gr. $\alpha \tilde{\imath} \xi \not \alpha \gamma \rho ı \varsigma^{160}$ ) bzw. das ganze Tier eben wegen der Form seiner Hörner auch eine hieroglyphische Lesung rnpt: „Jahr“ hatte, verband er kurzerhand die beiden Phänomene miteinander und machte das Horn des Steinbocks ( $b$ nisw) zu einer Neujahrsbezeichnung. Der Brauch, am Neujahrsfest Wüstenwild und damit Steinböcke zu opfern, mußte auf diese Weise für ihn noch einen zusätzlichen Sinn bekommen. Durch das Töten des Wüstenwildes und insbesondere des Steinbocks am Morgen vor dem Sothisaufgang konnte das Ge-

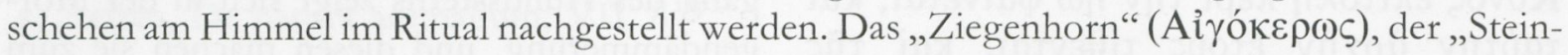
bock" neigte die Hörner und ging unter (er starb), im selben Augenblick töteten die Priester die Wüstentiere und ermöglichten so ritualhaft den kurz darauf folgenden Aufgang der Sothis. Die griechischen und lateinischen Autoren berichten von dem gleichen Ereignis, nur daß sie, weil sie wohl von dem astronomischen Zusammenhang und der Bezeichnung ${ }^{~} b$ ni $3 w$, die in Ägypten auf Edfu und Dendara beschränkt zu sein scheint, nichts wußten, statt des Steinbocks den gleichfalls am Neujahrsfest geopferten Oryx einsetzten. Das Anzeigen des Sothisaufgangs wurde dabei noch etwas verfremdet. Statt der Hörner des Tieres ist es jetzt sein Niesen [a, b] oder sein Schreien [c], astronomisch würde dies nicht allzuviel ändern, die Schnauzensterne o, $\pi$ und $\rho^{161}$ gingen etwa 5 min. vor den Sternen unter, die das Horn bilden ${ }^{162}$.

\section{Anhang: Die Identifikation des Tiers $t 3 w$ als $g h s:$ „Dorkasgazelle“}

Unter dem zu opfernden Wüstenwild taucht des öfteren auch ein Tier namens $t 3 w$ auf, dessen Identifikation bislang nicht geglückt ist. Das Wörterbuch $(V, 231,4)$ schreibt, daß es sich um ein Opfertier handele, das zusammen mit dem Steinbock erwähnt werde; vermutlich deswegen schlug Alliot „Mufflon (?)“ vor $^{163}$, das Fragezeichen ist dann bei Daumas verschwunden ${ }^{164}$. Betrachtet man die beigefügte Tabelle ${ }^{165}$, so stellt man fest, daß gḥs und $t 3 w$ für sich genommen

Dendarastellen sind D VII, 171, ult; 190,6-7; VIII, 104,13-4 und 105,11-2. Vgl. ebenfalls das Steinbock-, Gazellenund Oryxopfer in pWien 3865,13-4, Herbin, RdE 35, 1984, 108 und 125 sowie seine Bemerkungen auf S. 122-3.

157 $n h$, vgl. Edfou VI, 257,12.

${ }^{158}$ Siehe Ph. Derchain, Le sacrifice de l'oryx, Rites Égyptiens 1, Bruxelles, 1962.

${ }^{159}$ Der früheste bislang in Ägypten gefundene Tierkreis stammt von etwa 200 v. Chr., vgl. Ne u g eba u er-Pa rk e r, Astronomical Texts III, 204.

${ }^{160} \mathrm{Liddell} / \mathrm{S} \operatorname{cott}, \mathrm{S} .40$. Zoologisch ist damit meist die Bezoarziege (Paseng) gemeint, die eng mit dem echten Steinbock verwandt ist, vgl. Steier in RE, 2, Reihe, Bd. III, 2239 s. v. Steinbock.

${ }^{161}$ Vgl. Ptolemaios, ed. Manitius, Bd. II, 51.

${ }^{162}$ Überschlagsrechnungen nach P. V. Neugebauer, Astronomische Chronologie III, \$S 10 und 17.

${ }^{163}$ Le culte d'Horus à Edfou, 309 und 387.

${ }^{164}$ Mammisis, 211.

${ }^{165}$ Aufgenommen sind nur die Stellen, an denen mindestens drei Tiere des Wüstenwildes vorkommen, bisweilen sind die Determinative oder Ideogramme falsch bzw. ungenau (vgl. Edfou VI, 327 ult: „Oryx“ als Ideogramm für gḥs), was z. T. auch an den Editionen liegen wird. 
beliebig mit den Bezeichnungen für die Oryxantilope und den Steinbock kombinierbar sind, es jedoch kein einziges Mal vorkommt, daß ghs und $t 3 w$ gemeinsam in einer Inschrift genannt sind. Bei der Fülle der Belege geht die Wahrscheinlichkeit, daß dies Zufall ist, gegen Null, die Schlußfolgerung ist vielmehr die, daß gḥs und $t 3 w$ identisch sind. Besonders aufschlußreich ist hierfür Edfou VII, 164, wo im Titel (hrp 'wt nt ḩsst) gesagt wird, daß Oryxantilopen ( $m$ 3Dorkasgazellen (ghs) und Steinböcke ( $n i 3 w$ ) gefangen werden, jedoch der König in seiner zur Szene gehörenden Rede sagt: „Hiermit bringe ich dir Oryxantilopen ( $m h$ ) , Dorkasgazellen ( $t 3 w)$ und Steinböcke (nisw)“. D. h. t3w dürfte im selben Verhältnis zu gḥs stehen wie hnpp-wdist zu $m$ 3- $\underline{b} \underline{\underline{d}}$, die Etymologie bleibt jedoch im Unklaren ${ }^{166}$.

\begin{tabular}{|c|c|c|c|c|c|c|}
\hline \multirow[b]{2}{*}{ Stelle } & \multicolumn{2}{|c|}{ Gazelle } & \multicolumn{3}{|c|}{ Oryx } & \multirow{2}{*}{$\begin{array}{c}\text { Steinbock } \\
n i 3 w\end{array}$} \\
\hline & $g h s$ & $t 3 w$ & $m 3-\underline{b} \underline{d}$ & $m b$ & $h n p-w d \underline{d} s t$ & \\
\hline D IV $, 11,1-2$ & & $x$ & $x$ & & & $x$ \\
\hline D IV $, 22,8-9$ & $x$ & & $x$ & & & $\times$ \\
\hline D VI, 35,13-4 & $\times$ & & $x$ & & & $x$ \\
\hline D VII, 171, ult. & $x$ & & $x$ & & & $x$ \\
\hline D VII, 190,6-7 & $x$ & & $x$ & & & $\times$ \\
\hline D VIII, $104,13-4$ & $x$ & & $x$ & & & $\times$ \\
\hline D Mam., 24,7 & & $x$ & $x$ & & & $x$ \\
\hline D Mam., 231,5. & & $\times$ & & & $x$ & $x$ \\
\hline $\mathrm{E} \mathrm{I}^{2}, 452,4-5$ & $x$ & & $\times$ & & & $x$ \\
\hline $\mathrm{E} \mathrm{I} \mathrm{I}^{2}, 537,11$ & $x$ & & $\times$ & & & $x$ \\
\hline$E I^{2}, 549,5$ & $x$ & & $x$ & & & $x$ \\
\hline $\mathrm{E} \mathrm{I}^{2}, 553,15-6$ & & $x$ & $x$ & & & $x$ \\
\hline$E I^{2}, 555,9$ & & $x$ & - & & $x$ & $x$ \\
\hline E IV $, 65,1-2$ & $x$ & & $x$ & & & $x$ \\
\hline E IV, 351,1-2 & $x$ & & $x$ & & & $x$ \\
\hline E V $, 151,10$ & $x$ & & $x$ & & & $x$ \\
\hline E VI, 257,12-4 & & $x$ & & $x$ & & $x$ \\
\hline E VI, 327, ult. $-328,1$ & $x$ & & & $x$ & & $x$ \\
\hline E VII, 164,4 & $x$ & & $x$ & & & $x$ \\
\hline E VII, 164,8-9 & & $x$ & & $x$ & & $x$ \\
\hline E VII, 213,4-5 & $x$ & & $x$ & & & $\times$ \\
\hline E VII, 323,1 & & $x$ & & $x$ & & $x$ \\
\hline E VIII, 166,15 & $x$ & & $x$ & & & $x$ \\
\hline E VIII, 169,6-7 & $x$ & & $x$ & & & $x$ \\
\hline
\end{tabular}




\section{NACHTRAG ZU LEITZ, DIE NACHT DES KINDES IN SEINEM NEST IN DENDARA}

Nach Abschluß des Manuskripts erschien der Aufsatz von S. Cauville in BSFE 123, 1992, die ebenfalls bei der st kemt dšrt (sie schlägt noch etwas andere Lesungen vor, die aber an der Übersetzung nicht viel ändern) an den Sirius denkt, der seine Farbe von rot im Altertum zu blau in der heutigen Zeit verändert habe (vgl. oben Abschnitt IV). Sie zitiert dabei einen Artikel zweier französischer Astronomen, J. M. Bonnet-Bidaud und C. Gry, The stellar field in the vicinity of Sirius and the colour enigma in: Astronomy and Astrophysics 252, 1991, 193-7, die ihrerseits wieder eine ganze Reihe neuerer Lösungsvorschläge zu dem Farbproblem referieren. Ihr eigener Vorschlag ist, daß sich eine interstellare Wolke vor Sirius geschoben haben könnte, die eine temporäre Farbänderung bewirkt haben könnte. Sie berechnen dabei einen zeitweiligen Rückgang der scheinbaren Helligkeit des Sirius von $m=-1.5$ zu $m=+1.5$. Dieser Ansatz läßt sich mit dem gleichen Argument zurückweisen wie der von Schlosser, der eine scheinbare Helligkeit von etwa $\mathrm{m}=-4$ errechnete. Beide berücksichtigen nicht die dann nötige Änderung des arcus visionis (Sehungsbogen), der im einen Fall viel zu klein wäre (etwa $\left.6^{\circ} .2-6^{\circ} .3\right)$, im anderen viel zu groß (rund $12^{\circ} .5-13^{\circ}$ bei $\mathrm{m}=+1.5)$ und nicht mit den antiken Beobachtungen, die einen Wert von ungefähr $9^{\circ}$ nahelegen, korrelieren (vgl. oben Anm. 112). 